\title{
Classification of $(3+1)$ D Bosonic Topological Orders: The Case When Pointlike Excitations Are All Bosons
}

\author{
Tian Lan, ${ }^{1,2}$ Liang Kong, ${ }^{3}$ and Xiao-Gang Wen ${ }^{4}$ \\ ${ }^{1}$ Perimeter Institute for Theoretical Physics, Waterloo, Ontario N2L 2Y5, Canada \\ ${ }^{2}$ Institute for Quantum Computing, University of Waterloo, Waterloo, Ontario N2L $3 G 1$, Canada \\ ${ }^{3}$ Yau Mathematical Sciences Center, Tsinghua University, Beijing 100084, China \\ ${ }^{4}$ Department of Physics, Massachusetts Institute of Technology, Cambridge, Massachusetts 02139, USA
}

(Received 8 January 2018; revised manuscript received 20 April 2018; published 22 June 2018)

\begin{abstract}
Topological orders are new phases of matter beyond Landau symmetry breaking. They correspond to patterns of long-range entanglement. In recent years, it was shown that in $1+1 \mathrm{D}$ bosonic systems, there is no nontrivial topological order, while in $2+1 \mathrm{D}$ bosonic systems, the topological orders are classified by the following pair: a modular tensor category and a chiral central charge. In this paper, following a new line of thinking, we find that in $3+1 \mathrm{D}$ the classification is much simpler than it was thought to be; we propose a partial classification of topological orders for $3+1 \mathrm{D}$ bosonic systems: If all the pointlike excitations are bosons, then such topological orders are classified by a simpler pair $\left(G, \omega_{4}\right)$ : a finite group $G$ and its group 4-cocycle $\omega_{4} \in \mathcal{H}^{4}[G ; U(1)]$ (up to group automorphisms). Furthermore, all such $3+1 \mathrm{D}$ topological orders can be realized by Dijkgraaf-Witten gauge theories.
\end{abstract}

DOI: 10.1103/PhysRevX.8.021074

\section{INTRODUCTION}

Historically, large classes of matter states have only been completely classified a few times. The first was the classification of all spontaneous symmetry-breaking orders $[1,2]$. We find that all symmetry-breaking orders can be described by the pair

$$
\left(G_{\Psi} \subset G_{H}\right),
$$

where $G_{H}$ is the symmetry group of the system and $G_{\Psi}$, a subgroup of $G_{H}$, is the symmetry group of the ground state.

The second was the classification of all one-dimensional gapped quantum phases. We find that one-dimensional gapped quantum phases with on-site symmetry $G_{H}$ can be classified by a triple (even for strongly interacting bosons or fermions) $[3,4]$ :

$$
\left[G_{\Psi} \subset G_{H} ; \operatorname{pRep}\left(G_{\Psi}\right)\right]
$$

where $\operatorname{pRep}\left(G_{\Psi}\right)$ is a projective representation of $G_{\Psi}$ [5]. We see that all the one-dimensional gapped quantum phases are described by symmetry breaking plus an

Published by the American Physical Society under the terms of the Creative Commons Attribution 4.0 International license. Further distribution of this work must maintain attribution to the author(s) and the published article's title, journal citation, and DOI.
Subject Areas: Condensed Matter Physics,

Strongly Correlated Materials addition structure described by $\operatorname{pRep}\left(G_{\Psi}\right)$. The additional structure is the symmetry-protected topological (SPT) order [6]. The $\operatorname{pRep}\left(G_{\Psi}\right)$ structure can equivalently be described by a pair $\left(G_{\Psi}, \omega_{2}\right)$, where $\omega_{2}$ is a group 2-cocycle in $\mathcal{H}^{2}\left[G_{\Psi} ; U(1)\right]$.

The third classification is for two-dimensional gapped quantum phases. In the absence of any symmetry, a gapped phase may have a nontrivial topological order [7-9]. We find that all $2+1 \mathrm{D}$ bosonic topological orders are classified by a pair [9-11],

$$
(\mathrm{MTC}, c) \text {, }
$$

where MTC is a unitary modular tensor category and $c$ is the chiral central charge of the edge states. Physically, the tensor category theory MTC is just a theory that describes the fusion and the braiding of quasiparticle excitations, which correspond to fractional or non-Abelian statistics. Modular means that every nontrivial quasiparticle has a nontrivial mutual statistics with some quasiparticles.

The above idea of classification focused on the properties of topological excitations. Later, it was generalized to fermionic systems [12]. The classification then included symmetries $[13,14]$ and became a fairly complete classification. Traditionally, symmetries are described by groups, but even when we focus on only the excitations, no information of the symmetry is missing. This is because excitations carry representations of the symmetry group, from which the group can be uniquely reconstructed (known as the Tannaka duality). 
In particular, SPT orders $[15,16]$ in $2+1 \mathrm{D}$, which were known to be classified by the pair $\left(G, \omega_{3}\right)$, where $\omega_{3}$ are now group 3-cocycles, are also included in this complete classification as a special case. From the excitation point of view, they are equivalently classified by minimal modular extensions of symmetric fusion categories (SFC). Here, SFC describes the excitations above the symmetric product state, carrying group representations. Symmetric fusion emphasizes the fact that these excitations have trivial statistics: They are either bosons or fermions and have trivial mutual braidings. There are only two variants of SFC: One is $\mathcal{R e p}(G)$, the usual representation category, with all excitations being bosons; the other is $\operatorname{s} \mathcal{R e p}\left(G^{f}\right)$, for fermionic systems, with some representations assigned Fermi statistics. On the other hand, a minimal modular extension of the SFC just means the smallest MTC that contains the SFC. It corresponds to the gauged theory [17] of the SPT order, which is a topological order with no symmetry.

After the fairly complete classification results in $1+1 \mathrm{D}$ and $2+1 \mathrm{D}$, in this paper, we study the classification of $3+1 \mathrm{D}$ topological orders. We only study the simpler case, the $3+1 \mathrm{D}$ topological orders for bosonic systems. The $3+1 \mathrm{D}$ bosonic topological orders are gapped quantum liquids [18,19] without any symmetry. However, the physical definition of gapped quantum liquids, which is from the point of view of the renormalization group or local unitary transformation, is far from applicable. Instead, similar to the classification in $2+1 \mathrm{D}$, which focuses on the properties of topological excitations, we start with some basic properties of $3+1 \mathrm{D}$ topological orders:

(1) They have pointlike excitations and stringlike excitations that can move in all directions and have nontrivial braidings among them.

(2) The number of types of excitations is finite.

(3) The statistics of pointlike excitations is trivial; they are bosons or fermions with trivial mutual statistics. In other words, the pointlike excitations in a $3+1 \mathrm{D}$ topological order are described fully by a SFC.

(4) The braiding between pointlike and stringlike excitations, and among stringlike excitations must be nondegenerate, enough to detect every type of excitation. This is the principle of remote detectability, which will be elaborated on later.

(5) The $2+1 \mathrm{D}$ boundary of $3+1 \mathrm{D}$ topological order is always gappable. Furthermore, any interface or boundary induced by condensation of bosonic pointlike excitations is gappable. A detailed argument is given in Sec. VA.

(6) Just like 2+1D topological orders, the bulk $3+1 \mathrm{D}$ topological order is fully determined by its boundary (the so-called boundary-bulk duality [20,21]). Furthermore, one can generalize the Turaev-Viro TQFT [22] or Levin-Wen model [23] to construct the explicit model of bulk $3+1 \mathrm{D}$ topological order from its gapped boundary. Mathematically, this is the generalization of the Drinfeld center to higher dimensions.

One may view the above as our axioms or assumptions to mathematically define a $3+1 \mathrm{D}$ topological order. This excludes the gapped nonliquid states in $3+1 \mathrm{D}$, such as stacked fractional-quantum Hall layers [18], and fractal or fracton topological states [24-30], which include Haah's model [31].

If the pointlike excitations are all bosons, the corresponding SFC will always have the form $\operatorname{Rep}(G)$ for some finite group $G$. In other words, the pointlike excitations of a $3+1 \mathrm{D}$ topological order can always be viewed as carrying irreducible representations of the group [32]. They behave exactly like the quasiparticle excitations above a product state with $G$ symmetry. This is quite an amazing result: A $3+1 \mathrm{D}$ topological order whose quasiparticles are all bosonic is always related to a finite group $G$ [33]. We refer to such (pointlike-excitations-are-)all-boson topological order as $\mathrm{AB}$ topological order.

One may naturally wonder if a $3+1 \mathrm{D}$ AB topological order is always described by a $G$-gauge theory since the pointlike excitations in a $G$-gauge theory are indeed described by $\mathcal{R e p}(G)$. In fact, the above statement is not true. There are $3+1 \mathrm{D}$ topological orders arising from the Dijkgraaf-Witten gauge theory [34]. Dijkgraaf-Witten gauge theories can be viewed as gauged SPTs, which can be defined in any dimension. They are determined by, again, a pair $\left(G, \omega_{d+1}\right)$ : Here, $\omega_{d+1}$ is a group cocycle in $\mathcal{H}^{d+1}[G ; U(1)]$, where $d$ is the spatial dimension of the system. In $3+1 \mathrm{D}$, we need 4-cocycles. In a Dijkgraaf-Witten theory with $\left(G, \omega_{4}\right)$, pointlike excitations are also described by $\operatorname{Rep}(G)$. So we cannot say that all $3+1 \mathrm{D}$ topological orders with $\mathcal{R} \operatorname{ep}(G)$ pointlike excitations are described by the usual $G$-gauge theory. But, do we have something even more general than Dijkgraaf-Witten gauge theory that also produces $\mathcal{R} \operatorname{ep}(G)$ pointlike excitations? In this paper, we want to show that there is nothing more general:

All 3 + 1D topological orders, whose pointlike excitations are all bosons, are classified by a finite group $G$ and its group 4-cocycle $\omega_{4} \in \mathcal{H}^{4}[G ; U(1)]$, up to group automorphisms.

In this paper, "classified" always means a correspondence in a one-to-one fashion. The above result allows us to obtain a table of simple $3+1 \mathrm{D}$ AB topological orders (see Table I). Furthermore,

\section{All $3+1 \mathrm{D} A B$ topological orders can be realized by} Dijkgraaf-Witten gauge theory with a finite gauge group.

For a classification of $3+1 \mathrm{D}$ topological orders where some pointlike excitations are fermions, see Ref. [35].

The classification of $3+1 \mathrm{D} A B$ topological orders is obtained by condensing all the pointlike excitations in a $3+1 \mathrm{D}$ topological order $\mathcal{C}^{4}$ to form a new topological 
TABLE I. A table of simple $3+1 \mathrm{D}$ AB topological orders in bosonic systems, listed according to the number $N_{\text {exc }}$ of topological types for pointlike excitations. Here, $N_{\text {topAB }}$ is the number of distinct $\mathrm{AB}$ topological orders, and $\mathcal{H}_{\text {Aut }}^{4}(G)$ is the set of cocycles in $\mathcal{H}^{4}[G ; U(1)]$ up to the automorphisms of $G$ : $\mathcal{H}_{\text {Aut }}^{4}(G)=\mathcal{H}^{4}[G ; U(1)] /$ Aut. In particular, $\mathcal{H}^{4}\left[Z_{2} \times\right.$ $\left.Z_{2} ; U(1)\right]=\mathbb{Z}_{2}^{2}$ and $\mathcal{H}_{\text {Aut }}^{4}\left(Z_{2} \times Z_{2}\right)=\mathbb{Z}_{2}$.

\begin{tabular}{lccc}
\hline \hline$N_{\text {exc }}$ & $N_{\text {topAB }}$ & Classification: $\mathcal{H}_{\text {Aut }}^{4}(G)$ & Examples \\
\hline 1 & 1 & $\mathcal{H}_{\text {Aut }}^{4}\left(Z_{1}\right)=\mathbb{Z}_{1}$ & Trivial product states \\
2 & 1 & $\mathcal{H}_{\text {Aut }}^{4}\left(Z_{2}\right)=\mathbb{Z}_{1}$ & $Z_{2}$ gauge theory \\
3 & 2 & $\mathcal{H}_{\text {Aut }}^{4}\left(Z_{3}\right)=\mathbb{Z}_{1}$, & $Z_{3}$ and $S_{3}$ gauge \\
& & $\mathcal{H}_{\text {Aut }}^{4}\left(S_{3}\right)=\mathbb{Z}_{1}$ & theories \\
4 & 5 & $\mathcal{H}_{\text {Aut }}^{4}\left(Z_{4}\right)=\mathbb{Z}_{1}$, & \\
& & $\mathcal{H}_{\text {Aut }}^{4}\left(Z_{2} \times Z_{2}\right)=\mathbb{Z}_{2}$, & \\
& & $\mathcal{H}_{\text {Aut }}^{4}\left(D_{10}\right)=\mathbb{Z}_{1}$, & \\
$\ldots$ & $\ldots$ & $\mathcal{H}_{\text {Aut }}^{4}\left(A_{4}\right)=\mathbb{Z}_{1}$ \\
\hline \hline
\end{tabular}

order $\mathcal{D}^{4}$ (which is possible when all the pointlike excitations are bosons). We make the following arguments.

(1) The new phase $\mathcal{D}^{4}$ is a trivial phase. Therefore, $\mathcal{C}^{4}$ has a $2+1 \mathrm{D}$ gapped boundary $\mathcal{M}^{3}$ induced by such condensation, which carries only stringlike excitations.

(2) The above stringlike excitations on the boundary are labeled by the elements of a finite group $G$, and their fusion rule is given by the group multiplication. It is the same group whose representations are carried by the pointlike excitations in the bulk.

(3) The string-only boundary $\mathcal{M}^{3}$ forms a unitary pointed fusion 2-category whose only nontrivial level are the objects. The different pointed fusion 2-categories are classified by a finite group $G$ and its 4-cocycle $\omega_{4}$ in $\mathcal{H}^{4}(G ; U(1))$, up to group automorphisms.

(4) The bulk topological order $\mathcal{C}^{4}$ is the center of the fusion 2-category $\mathcal{M}^{3}: \mathcal{C}^{4}=Z\left(\mathcal{M}^{3}\right)$ [20,21], which is a Dijkgraaf-Witten gauge theory with $\left(G, \omega_{4}\right)$. Furthermore, each bulk topological order $\mathcal{C}^{4}$ corresponds to a unique unitary pointed fusion 2-category $\mathcal{M}^{3}$.

In the following, we discuss some general properties of $3+1 \mathrm{D}$ topological orders. Then, we show the main result of the paper following the above four steps.

\section{EXCITATIONS IN TOPOLOGICALLY ORDERED STATES}

\section{A. Pointlike excitations}

\section{Using the trap Hamiltonian to define excitations}

Consider a bosonic system defined by a local gapped Hamiltonian $H_{0}$ in $d$-dimensional space $M^{d}$ without a boundary. A collection of quasiparticle excitations labeled by $p_{i}$ and located at $\mathbf{x}_{\mathbf{i}}$ can be produced as gapped ground states of $H_{0}+\sum_{i} \delta H_{p_{i}}$, where $\delta H_{p_{i}}$ is nonzero only near $\mathbf{x}_{\mathbf{i}}$. By choosing different $\delta H_{p_{i}}$ 's we can create (or trap) all kinds of pointlike excitations. The gapped ground states of $H_{0}+\sum_{i} \delta H_{p_{i}}$ may have a degeneracy $D\left(M^{d} ; p_{1}, p_{2}, \ldots\right)$, which depends on the quasiparticle types $p_{1}, p_{2}, \ldots$ and the topology of the space $M^{d}$. The degeneracy is not exact, but it becomes exact in the large-space and large-particle separation limit. We use $\mathcal{V}\left(M^{d} ; p_{1}, p_{2}, \ldots\right)$ to denote the space of the degenerate ground states, which will also be called fusion space. If the Hamiltonian $H_{0}+\sum_{i} \delta H_{p_{i}}$ is not gapped, we say $D\left(M^{d} ; p_{1}, p_{2}, \ldots\right)=0$ [i.e., $\mathcal{V}\left(M^{d} ; p_{1}, p_{2}, \ldots\right)$ has zero dimension]. If $H_{0}+\sum_{i} \delta H_{p_{i}}$ is gapped, but if $\delta H_{p_{i}}$ also creates quasiparticles away from the $\mathbf{x}_{\mathbf{i}}$ 's (indicated by the bump in the energy density away from the $\mathbf{x}_{\mathbf{i}}$ 's), we also say $D\left(M^{d} ; p_{1}, p_{2}, \ldots\right)=0$. (In this case, quasiparticles at the $\mathbf{x}_{\mathbf{i}}$ 's do not fuse to trivial quasiparticles.) So, if $D\left(M^{d} ; p_{1}, p_{2}, \ldots\right)>0, \delta H_{p_{i}}$ only creates or traps quasiparticles at the $\mathbf{x}_{\mathbf{i}}$ 's.

For topologically ordered states with no spontaneous symmetry breaking, the fusion space on a $d$-dimensional sphere $M^{d}=S^{d}$ with no particles $\mathcal{V}\left(S^{d}\right)$ is always one dimensional. Thus, in the presence of pointlike excitations, the dimension of the fusion space, $\mathcal{V}\left(S^{d} ; p_{1}, p_{2}, \ldots\right)$, represents the total number of internal degrees of freedom for the quasiparticles $p_{1}, p_{2}, \ldots$. To obtain the number of internal degrees of freedom for type- $p_{i}$ quasiparticles, we consider the dimension $D\left(S^{d} ; p_{i}, p_{i}, \ldots, p_{i}\right)$ of the fusion space on $n$ type- $p_{i}$ particles on $S^{d}$. In the large- $n$ limit, $D\left(S^{d} ; p_{i}, p_{i}, \ldots, p_{i}\right)$ has the form

$$
\ln D\left(S^{d} ; p_{i}, p_{i}, \ldots, p_{i}\right)=n\left(\ln d_{p_{i}}+o(1 / n)\right) .
$$

Here, $d_{p_{i}}$ is called the quantum dimension of the type- $p_{i}$ particle, which describes the internal degrees of freedom of the particle. For example, a spin-0 particle has a quantum dimension $d=1$, while a spin-1 particle has a quantum dimension $d=3$.

\section{Simple type and composite type}

Two excitations $p$ (trapped by $\Delta H_{p}$ ) and $p^{\prime}$ (trapped by $\left.\Delta H_{p^{\prime}}\right)$ are said to have the same type if the corresponding fusion spaces $\mathcal{V}\left(S^{d} ; p, p_{1}, \ldots\right)$ and $\mathcal{V}\left(S^{d} ; p^{\prime}, p_{1}, \ldots\right)$ can smoothly deform into each other as we change the trap Hamiltonian from $\Delta H_{p}$ to $\Delta H_{p^{\prime}}$. Two excitations $p$ and $p^{\prime}$ are of the same type iff they only differ by some local operators. If an excitation can be created by local operators from the ground state, the excitation is said to have a trivial type and is denoted as $\mathbf{1}$.

Even after we quotient out the local excitations of trivial type, there are still two kinds of topological quasiparticle types: the simple type and composite type. If the ground-state degenerate subspace $\mathcal{V}\left(M^{d} ; p, q, \ldots\right)$ cannot be split by any small local perturbations near $\Delta H_{p}$, 
then the particle $p$ is said to be simple. Otherwise, the particle type $p$ is said to be composite.

When $p$ is composite, the fusion space $\mathcal{V}\left(M^{d} ; p, q, \ldots\right)$ has a direct sum decomposition (after splitting by a generic perturbation of $\Delta H_{p}$ ):

$$
\begin{aligned}
\mathcal{V}\left(M^{d} ; p, q, \ldots\right)= & \mathcal{V}\left(M^{d} ; p_{1}, q, \ldots\right) \oplus \mathcal{V}\left(M^{d} ; p_{2}, q, \ldots\right) \\
& \oplus \mathcal{V}\left(M^{d} ; p_{3}, q \ldots\right) \oplus \ldots,
\end{aligned}
$$

where $p_{1}, p_{2}, p_{3}$, etc. are simple types. The above decomposition allows us to denote the composite type $i_{1}$ as

$$
p=p_{1} \oplus p_{2} \oplus p_{3} \oplus \ldots
$$

\section{Fusion of pointlike excitations}

When we fuse two simple types of topological particles $p_{1}$ and $p_{2}$ together, they may become a topological particle of a composite type:

$$
p_{1} \otimes p_{2}=q=p_{3}^{\prime} \oplus p_{3}^{\prime \prime} \oplus \ldots
$$

Here, we use an integer tensor $N_{p_{3}}^{p_{1} p_{2}}$ to describe the quasiparticle fusion, where $p_{i}$ label simple types:

$$
p_{1} \otimes p_{2}=\bigoplus_{p_{3}} N_{p_{3}}^{p_{1} p_{2}} p_{3} .
$$

Such an integer tensor $N_{p_{3}}^{p_{1} p_{2}}$ is referred to as the fusion coefficients of the topological order, which is a universal property of the topologically ordered state.

The internal degrees of freedom (i.e., the quantum dimension $d_{p}$ ) for the type- $p$ simple particle can be calculated directly from $N_{p_{3}}^{p_{1} p_{2}}$. In fact, $d_{p}$ is the largest eigenvalue of the matrix $N_{p}$, whose elements are $\left(N_{p}\right)_{p_{2} p_{1}}=N_{p_{2}}^{p p_{1}}$.

\section{B. Stringlike excitations}

Similarly, we can also use gapped trap Hamiltonians $H_{0}+\sum_{s} \Delta H_{s}$ to define stringlike excitations, where $\Delta H_{s}$ is nonzero only near a loop. The ground-state subspace of $H_{0}+\sum_{s} \Delta H_{s}$ is called the fusion space of strings $\mathcal{V}\left(M^{d}, s, t, \ldots\right)$. If the fusion spaces $\mathcal{V}\left(M^{d}, s, t, \ldots\right)$ and $\mathcal{V}\left(M^{d}, s^{\prime}, t, \ldots\right)$ can smoothly deform into each other, we say the strings $s$ and $s^{\prime}$ are of the same type.

If the ground-state degenerate subspace $\mathcal{V}\left(M^{d} ; s, t, \ldots\right)$ cannot be split by any small nonlocal perturbations along the string $s$, then the string $s$ is said to be simple. Otherwise, the string $s$ is said to be composite. We stress that, here, we allow nonlocal perturbations along the string $s$. In other words, any degrees of freedom near the string can interact no matter how far away they are. But the interactions do not involve degrees of freedom far away from the string. Nonlocal perturbations are necessary. If we use local perturbations to define string types, we would have too many string types that are not related to the topological orders in the ground state.

The fusion of the string loops is also described by integer tensors,

$$
s_{1} \otimes s_{2}=\bigoplus_{s_{3}} M_{s_{3}}^{s_{1} s_{2}} s_{3}
$$

The string loops can also shrink and become pointlike excitations,

$$
s_{i} \rightarrow \bigoplus_{p_{j}} M_{p_{j}}^{s_{i}} p_{j} .
$$

We like to conjecture that,

if $M_{1}^{s}>1$, then the string $s$ is not simple (i.e., $s$ is a direct sum of several strings).

If a simple string satisfies $M_{1}^{s}=1$, we say that $s$ is a pure simple string.

\section{On-string excitations are always gappable}

The strings, as 1D extended objects, may carry excitations that travel along them. Those excitations can be gapless at times. In the following, we argue that, if the types of pointlike and stringlike excitations are finite, the onstring excitations can always be gapped by adding proper interactions.

We make this argument by contradiction. Assuming that on-string excitations cannot be gapped by interactions, based on what we know about $1+1 \mathrm{D}$ systems, there are only two situations.

(1) When the on-string excitations are chiral with a nonzero chiral central charge $c$. In this case, when fusing $n$ strings together, the new string will have on-string excitations with chiral central charge $n c$. This means that the fusion will produce infinite types of strings. The finite string-type assumption excludes the possibility of on-string excitations with a nonzero chiral central charge.

(2) When the on-string excitations are described by the edge of certain fractional-quantum Hall states (which have chiral central charge $c=0$ ) [36]. In this case, the on-string excitations have a gravitational anomaly described by a noninvertible $2+1 \mathrm{D}$ topological order [20,37]. The open membrane operator that creates such a string on its boundary must creates a noninvertible $2+1 \mathrm{D}$ topological order on the membrane. Multiplying membrane operators corresponds to stacking $2+1 \mathrm{D}$ topological orders together, and stacking noninvertible topological orders can never produce a trivial topological order [20]. Thus, fusing $n$ strings together will always produce a new nontrivial string. Again, finite string-type assumptions exclude this possibility.

We remark that ungappable strings can appear in gapped nonliquid states $[18,19]$, such as the $3+1 \mathrm{D}$ gapped states 
obtained by stacked fractional-quantum Hall layers. But in that case, the strings are not mobile in all directions. It appears that the liquid assumption of topological order $[18,19]$ makes all strings gappable in $3+1 \mathrm{D}$. In the rest of this paper, we always assume that the on-string excitations are gapped.

\section{SOME GENERAL PROPERTIES OF 3 + 1D TOPOLOGICAL ORDERS OF BOSON SYSTEMS}

\section{A. Group structure in 3 + 1D topological order}

We note that the pointlike excitations in $3+1 \mathrm{D}$ topological orders are described by a SFC. Physically, a SFC is just a collection of particles, which are all bosons or fermions with trivial mutual statistics.

Mathematically, it has been shown that a SFC must be either $\operatorname{Rep}(G)$ [a braided fusion category (BFC) formed by the representations of $G$, with all the irreducible representations being assigned Bose statistics] or $\mathrm{s} \mathcal{R e p}(G)$ (a BFC formed by the representations of $G$, with some of the irreducible representations being assigned Bose statistics and other irreducible representations being assigned Fermi statistics) for some group $G$. Such a group $G$ is uniquely determined (up to isomorphisms) by the SFC, namely, by the pointlike excitations. This is known as the Tannaka duality, which will be further explained in Sec. V C.

The above implies that

each 3+1D topological order is associated with a group $G$, where the pointlike excitations (particles) are described by $\mathcal{R e p}(G)$ or $\operatorname{s} \mathcal{R e p}(G)$.

In this paper, we use this fact to gain a systematic understanding of $3+1 \mathrm{D}$ topological orders. (In fact, each higher-dimensional topological order is also related to a group in the same fashion.) In some sense, 3+1D topological orders can all be viewed as gauge theories with some old or new twists.

However, those pointlike excitations have trivial mutual statistics among them. One cannot use the pointlike excitations to detect other pointlike excitations by remote operations. In general, we believe $[20,36]$ in the following

"principle of remote detectability": In an anomaly-free topological order, every topological excitation can be detected by other topological excitations via some remote operations; therefore, the topological order is anomaly-free.

Here "anomaly-free" means realizable by a local bosonic model on a lattice in the same dimension [37]. Thus, the remote detectability condition is also the anomaly-free condition.

The above principle implies that an anomaly-free (i.e., realizable) $3+1 \mathrm{D}$ topological order must contain stringlike topological excitations; thus, every pointlike topological excitation can be detected by some stringlike topological excitations via remote braiding, and every stringlike topological excitation can be detected by some pointlike and/or stringlike topological excitations via remote braiding. We see that the properties of stringlike topological excitations are determined by the pointlike topological excitations [i.e., $\mathcal{R} \operatorname{ep}(G)$ or $\mathrm{s} \mathcal{R} \operatorname{ep}(G)$ ] to a certain degree.

\section{B. Dimension reduction of topological orders}

To better understand the relation between the pointlike and stringlike excitations, we introduce the dimension reduction in the next section, which turns out to be a very useful tool in our approach. We can reduce a $d+1 \mathrm{D}$ topological order $\mathcal{C}^{d+1}$ on space-time $M^{d} \times S^{1}$ to $d \mathrm{D}$ topological orders on space-time $M^{d}$ by making the circle $S^{1}$ small (see Fig. 1). In this limit, the $d+1 \mathrm{D}$ topological order $\mathcal{C}^{d+1}$ can be viewed as several $d \mathrm{D}$ topological orders $\mathcal{C}_{i}^{d}, i=1,2, \ldots, N_{1}^{\mathrm{sec}}$, which happen to have degenerate ground-state energy. We denote such a dimensional reduction process by

$$
\mathcal{C}^{d+1}=\bigoplus_{i=1}^{N_{1}^{\mathrm{sec}}} \mathcal{C}_{i}^{d}
$$

where $N_{1}^{\mathrm{sec}}$ is the number of sectors produced by the dimensional reduction.

For example, let us use $\mathcal{C}_{G}^{d+1}$ to denote the $d+1 \mathrm{D}$ topological order described by gauge theory with the finite gauge group $G$. We find that, for $d \geq 3$ (see Table II) [38,39],

$$
\mathcal{C}_{G}^{d+1}=\bigoplus_{\chi} \mathcal{C}_{G_{\chi}}^{d},
$$

where $\bigoplus_{\chi}$ sums over all different conjugacy classes $\chi$ of $G$, and $G_{\chi}$ is a subgroup of $G$ formed by all the elements that commute with an element in $\chi$. In fact, each dimensionreduced $d \mathrm{D}$ topological order $\mathcal{C}_{G_{\chi}}^{d}$ is produced by threading a $G$-gauge flux described by the conjugacy classes $\chi$ through $S^{1}$ in the space-time $M^{d} \times S^{1}$. The $\chi$ flux breaks the gauge symmetry $G$ down to $G_{\chi}$. Thus, the corresponding $d \mathrm{D}$ topological order is a $G_{\chi}$-gauge theory.

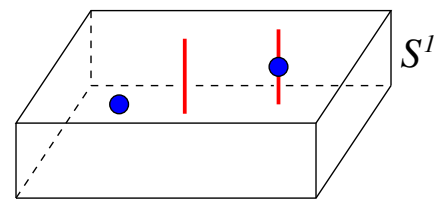

FIG. 1. The dimension reduction of $3 \mathrm{D}$ space $M^{2} \times S^{1}$ to $2 \mathrm{D}$ space $M^{2}$. The top and bottom surfaces are identified, and the vertical direction is the compactified $S^{1}$ direction. A 3D pointlike excitation (blue dot) becomes an anyon particle in 2D. A 3D stringlike excitation wrapping around $S^{1}$ (red line) also becomes an anyon particle in $2 \mathrm{D}$. 
TABLE II. The dimension reduction $M^{3} \times S^{1}$ to $M^{3}$ of the $3+1 \mathrm{D} S_{3}$-gauge theory $\mathcal{C}_{S_{3}}^{4}$, where $G_{\chi}$ 's are $S_{3}, Z_{2}, Z_{3}$. The $3+1 \mathrm{D}$ pointlike excitations $p_{0}, p_{1}, p_{2}$ become the $2+1 \mathrm{D}$ pointlike excitations. The $3+1 \mathrm{D}$ looplike excitations $s_{\chi q}$, when wrapped around $S_{1}$, also become the $2+1 \mathrm{D}$ pointlike excitations. Here, $\mathcal{C}_{S_{3}}^{3}$ is the untwisted sector, where $\mathbf{1}, A^{1}, A^{2}$ correspond to $\operatorname{Rep}\left(S_{3}\right)$. See Ref. [38] and Appendix A.

\begin{tabular}{lccc}
\hline \hline $\mathcal{C}_{S_{3}}^{4} \rightarrow$ & $\mathcal{C}_{S_{3}}^{3}$ & $\mathcal{C}_{Z_{2}}^{3}$ & $\mathcal{C}_{Z_{3}}^{3}$ \\
\hline Symmetry breaking & $S_{3} \rightarrow S_{3}$ & $S_{3} \rightarrow \mathbb{Z}_{2}$ & $S_{3} \rightarrow \mathbb{Z}_{3}$ \\
$p_{0} \rightarrow$ & & 1 & 1 \\
$p_{1} \rightarrow$ & $A^{1}$ & $e$ & 1 \\
$p_{2} \rightarrow$ & $A^{2}$ & $\boldsymbol{1} \oplus e$ & $e_{1} \oplus e_{2}$ \\
$s_{20} \rightarrow$ & $B$ & $\mathrm{~m}$ & $\cdots$ \\
$s_{21} \rightarrow$ & $B^{1}$ & $\mathrm{em}$ & $\cdots$ \\
$s_{30} \rightarrow$ & $C$ & $\cdots$ & $m_{1} \oplus m_{2}$ \\
$s_{31} \rightarrow$ & $C^{1}$ & $\cdots$ & $e_{1} m_{1} \oplus e_{1} m_{2}$ \\
$s_{32} \rightarrow$ & $C^{2}$ & $\cdots$ & $e_{2} m_{1} \oplus e_{2} m_{2}$ \\
\hline \hline
\end{tabular}

For Dijkgraaf-Witten theories (gauge theories twisted by group cocycles of the gauge group), dimension reduction has the form [39]

$$
\mathcal{C}_{G, \omega_{d+1}^{G}}^{d+1}=\bigoplus_{\chi} \mathcal{C}_{G_{\chi}, \omega_{d}^{G_{\chi}}(\chi)},
$$

where $\omega_{d+1}^{G}$ is a $(d+1)$-group cocycle $\omega_{d+1}^{G} \in \mathcal{H}^{d+1}$ $(G ; U(1)), \quad \omega_{d}^{G_{\chi}}(\chi)$ is a $d$-group cocycle $\omega_{d}^{G_{\chi}}(\chi) \in$ $\mathcal{H}^{d}\left(G_{\chi} ; U(1)\right)$, and $\mathcal{C}_{G, \omega_{d+1}^{G}}^{+1}$ is the topological order described by Dijkgraaf-Witten theory, with gauge group $G$ and cocycle twist $\omega_{d+1}^{G}$.

To understand the number of sectors $N_{1}^{\mathrm{sec}}$ in the dimension reduction, we note that the different sectors come from the different holonomy of moving pointlike excitations around $S^{1}$ (see Fig. 1). For gauge theory, this so-called holonomy comes from the gauge flux going through the compactified $S^{1}$. For more general topological orders, this holonomy comes from threading codimension2 topological excitations through $S^{1}$.

From this picture, we see that the number of sectors, $N_{1}^{\mathrm{sec}}$, is bounded by the number of types of codimension- 2 pure topological excitations. Also, if two codimension-2 topological excitations cannot be distinguished by their braiding with pointlike excitations, then threading them through $S^{1}$ will not produce different sectors. Thus, the number of sectors, $N_{1}^{\mathrm{sec}}$, is the number of classes of codimension-2 topological excitations that can be distinguished by braiding with the pointlike excitations. In particular, in $3+1 \mathrm{D}, N_{1}^{\mathrm{sec}}$ is the number of classes of stringlike topological excitations that can be distinguished by braiding with the pointlike excitations.

From the above discussion, we also see that the dimension reduction always contains a sector where we do not thread any nontrivial string through $S^{1}$ and where the holonomy of moving any pointlike excitations around $S^{1}$ is trivial. Such a sector is called the untwisted sector. Also, if a topological order has no nontrivial pointlike excitations, then its dimension reduction contains only one sector-the untwisted sector.

In the untwisted sector, there are three kinds of anyons. The first kind of anyon corresponds to the $3+1 \mathrm{D}$ pointlike excitations. The second kind of anyon corresponds to the $3+1 \mathrm{D}$ pure stringlike excitations wrapping around the compactified $S^{1}$. The third kind of anyon includes bound states of the first two kinds (see Fig. 1).

We point out that the untwisted sector in the dimension reduction can even be realized directly in 3D space without compactification. Consider a 2D submanifold in 3D space (see Fig. 2), and put the 3D pointlike excitations on the 2D submanifold. We can have a loop of string across the 2D submanifold, which can be viewed as an effective pointlike excitation on the 2D submanifold. We can also have a bound state of the above two types of effective pointlike excitations on the 2D submanifold. Those effective pointlike excitations on the 2D submanifold can fuse and braid just like the anyons in $2+1 \mathrm{D}$. The principle of remote detectability requires those effective pointlike excitations to form a MTC. When we perform dimension reduction, the above MTC becomes the untwisted sector of the dimension reduced $2+1 \mathrm{D}$ topological order. We mention that the dimension reduction introduces new types of perturbations that may not be local from the $3+1 \mathrm{D}$ point of view. But those new perturbations are local in the dimension-reduced $2+1 \mathrm{D}$ theory. MTC is very rigid, which cannot be changed by any $2+1 \mathrm{D}$ local perturbations. This is why the untwisted sector is still described by the same MTC that describes the effective pointlike excitations on the $2 \mathrm{D}$ submanifold.

Thus, we show that

the distinct (simple) pointlike excitations in the $3+1 \mathrm{D}$ topological order become the distinct anyons (i.e., the simple objects) in the dimension-reduced $2+1 \mathrm{D}$ topological order that corresponds to the untwisted sector.

Table II describes the dimension reduction of a $3+1 \mathrm{D}$ topological order $\mathcal{C}_{S_{3}}^{4}$ described by $S_{3}$-gauge theory:

$$
\mathcal{C}_{S_{3}}^{4} \rightarrow \mathcal{C}_{S_{3}}^{3} \oplus \mathcal{C}_{Z_{2}}^{3} \oplus \mathcal{C}_{Z_{3}}^{3} .
$$

The $2+1 \mathrm{D}$ topological order $\mathcal{C}_{S_{3}}^{3}$ is the untwisted sector. The three types of particles in $3+1 \mathrm{D} \mathcal{C}_{S_{3}}^{4}-p_{0}, p_{1}$,

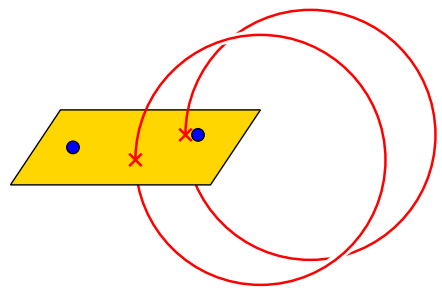

FIG. 2. The untwisted sector in the dimension reduction can be realized directly on a $2 \mathrm{D}$ submanifold in $3 \mathrm{D}$ space without compactification. 
$p_{2}$-that form a $\operatorname{SFC} \operatorname{Rep}\left(S_{3}\right)$ become three types of particles in the untwisted sector $\mathcal{C}_{S_{3}}^{3}-\mathbf{1}, A^{1}, A^{2}$ - that also form a $\operatorname{Rep}\left(S_{3}\right)$. We also see that in other sectors, the distinct pointlike excitations in 3+1D may not be reduced to distinct simple objects in the dimension-reduced $2+1 \mathrm{D}$ topological orders.

Since the dimension-reduced $2+1 \mathrm{D}$ topological orders must be anomaly-free, they are described by the modular tensor category. Since the untwisted sector always contains $\mathcal{R e p}(G)$, we conclude that

the untwisted sector of a dimension-reduced $3+1 \mathrm{D}$ topological order is a modular extension of $\mathcal{R} \operatorname{ep}(G)$.

In the next section, we show that such a modular extension must be a minimal one.

\section{Untwisted sector of dimension reduction is the Drinfeld center of $\mathcal{E}$}

In the following, we show a stronger result for the untwisted sector. Given a $3+1 \mathrm{D}$ bosonic topological order, let the symmetric fusion category formed by the pointlike excitations be $\mathcal{E}, \mathcal{E}=\mathcal{R} \operatorname{ep}(G)$ or $\mathcal{E}=\operatorname{s} \mathcal{R e p}\left(G^{f}\right)$.

The untwisted sector $\mathcal{C}_{\mathrm{untw}}^{3}$ of the dimension reduction of $3+1 \mathrm{D}$ topological orders must be the 2+1D topological order described by the Drinfeld center of $\mathcal{E}$ : $\mathcal{C}_{\text {untw }}^{3}=Z(\mathcal{E})$.

Note that the Drinfeld center $Z(\mathcal{E})$ is the minimal modular extension of $\mathcal{E}$.

First, let us recall the definition of the Drinfeld center. The Drinfeld center $Z(\mathcal{A})$ of a fusion category $\mathcal{A}$ is a braided fusion category, whose objects are pairs $\left(A, b_{A,-}\right)$, where $A$ is an object in $\mathcal{A}$ and $b_{A,-}$ is a set of isomorphisms $b_{A, X}: A \otimes X \cong X \otimes A, \forall X \in \mathcal{A}$, satisfying natural conditions. Note that $b_{A, X}$ is called a half-braiding. Morphisms between the pairs $\left(A, b_{A,-}\right),\left(B, b_{B,-}\right)$ are a subset of morphisms between $A, B$, such that they commute with the half-braidings $b_{A,-}, b_{B,-}$. The fusion and braiding of pairs is given by

$$
\begin{aligned}
\left(A, b_{A,-}\right) \otimes\left(B, b_{B,-}\right) & =\left(A \otimes B,\left(b_{A,-} \otimes i d_{B}\right)\left(i d_{A} \otimes b_{B,-}\right),\right. \\
c_{\left(A, b_{A,-}\right),\left(B, b_{B,-}\right)} & =b_{A, B} .
\end{aligned}
$$

In other words, to half-braid $A \otimes B$, one just half-braids $B$ and $A$ successively, and the braiding between pairs is nothing but the half-braiding.

Here, $\mathcal{C}_{\text {untw }}^{3}=Z(\mathcal{E})$ is a consequence of the fact that the strings in the untwisted sectors are in fact shrinkable. From the effective theory point of view, we can shrink a string $s$ (including bound states of particles with strings, in particular, pointlike excitations viewed as bound states with the trivial string) to a pointlike excitation $p_{s}^{\text {shr }}$ in $\mathcal{E}$,

$$
s \rightarrow p_{s}^{\mathrm{shr}}=p_{1} \oplus p_{2} \oplus \ldots, \quad p_{1}, p_{2}, \ldots \in \mathcal{E} .
$$

So if we only consider fusion, the particles $s, p$ in the dimension-reduced untwisted sector $\mathcal{C}_{\text {untw }}^{3}$ can all be viewed as the particles in $\mathcal{E}$, regardless of whether they come from the $3 \mathrm{D}$ particles or $3 \mathrm{D}$ strings. In particular, the particles from the $3+1 \mathrm{D}$ strings $s$ can be viewed as composite particles in $\mathcal{E}$ [see Eq. (16)]. To obtain the Drinfeld center, we need to introduce braiding among particles $p$ and $s$.

In the untwisted sector, the braiding between strings $s, s^{\prime}$, denoted by $c_{s, s^{\prime}}$, requires that string $s^{\prime}$ moves through string $s$, which prohibits shrinking of string $s$. However, there is no harm in considering the shrinking if we focus on only the initial and end states of the braiding process.

In particular, the braiding between a string $s$ and a particle $p$ induces an isomorphism between the initial and end states where the string $s$ shrinks (see Fig. 3),

$$
c_{s, p}^{\mathrm{shr}}: p_{s}^{\mathrm{shr}} \otimes p \cong p \otimes p_{s}^{\mathrm{shr}},
$$

which is automatically a half-braiding on the particle $p_{s}^{\text {shr }}$. Thus, $\left(p_{s}^{\mathrm{shr}}, c_{s,-}^{\mathrm{shr}}\right)$, by definition, is an object in the Drinfeld center $Z(\mathcal{E})$. Shrinking induces a functor

$$
\begin{aligned}
\mathcal{C}_{\text {untw }}^{3} & \rightarrow Z(\mathcal{E}), \\
s & \mapsto\left(p_{s}^{\mathrm{shr}}, c_{s,-}^{\mathrm{shr}}\right),
\end{aligned}
$$

which is obviously monoidal and braided; i.e., it preserves fusion and braiding. It is also fully faithful, namely, bijective on the morphisms. Physically, this means that the local operators on both sides are the same. On the left side, morphisms on a string $s$ are operators acting near (local to) the string $s$; on the right side, morphisms in the Drinfeld center are morphisms on the particle $p_{s}^{\text {shr }}$, which commute with the half-braiding $c_{s,-}^{\text {shr }}$. From the shrinking picture, morphisms on $p_{s}^{\text {shr }}$ can be viewed as operators acting both near the string $s$ and on the interior of the string (namely, on a disk $D^{2}$ ). But in order to commute with $c_{s, p}$ for all $p$, which can be represented by string operators for all $p$ going through the interior of the string $s$ (this includes
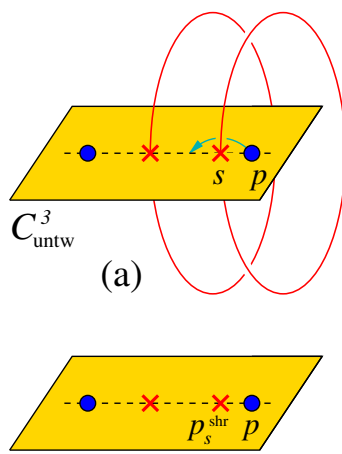

(c)
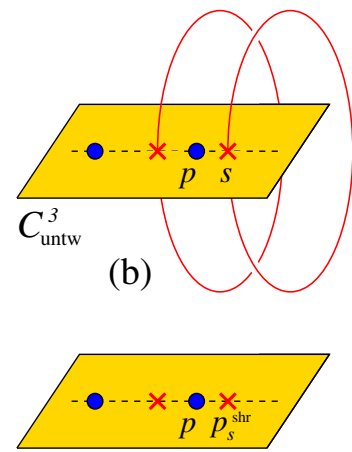

(d)
FIG. 3. (a,b) Braiding $c_{s, p}$ in the untwisted sector. Diagrams (c) and (d) are obtained from diagrams (a) and (b) by shrinking the strings. Shrinking thus induces a "half-braiding" isomorphism $c_{s, p}^{\mathrm{shr}}$ from (c) to (d). 
all possible string operators because string operators for all particles form a basis), we can take only the operators that act trivially on the interior of the string. Therefore, morphisms on the right side are also operators acting only near the string. This establishes that the functor is fully faithful and thus a braided monoidal embedding functor; in other words, $\mathcal{C}_{\text {untw }}^{3}$ can be viewed as a full sub-MTC of $Z(\mathcal{E})$. However, $Z(\mathcal{E})$ is already a minimal modular extension of $\mathcal{E}$, which implies that

$$
\mathcal{C}_{\text {untw }}^{3}=Z(\mathcal{E}) .
$$

As $Z(\mathcal{E})$ is well known, many properties can be easily extracted. For example, objects in $Z(\mathcal{E})$ have the form $(\chi, \rho)$, where $\chi$ is a conjugacy class and $\rho$ is a representation of the subgroup that centralizes $\chi$. One then makes the following conclusions:

(1) A looplike excitation in a 3 $+1 \mathrm{D}$ topological order always has an integer quantum dimension, which is $|\chi| \operatorname{dim} \rho$. (2) Pure strings ( $\rho$ trivial) always correspond to conjugacy classes of the group.

We also see that a $3+1 \mathrm{D}$ bosonic topological order is similar to a gauge theory of a finite group $G$. The following properties are the same: (1) the quantum dimensions of pointlike and stringlike excitations, (2) the fusion rule of those excitations, and (3) particle-loop and two-loop braidings.

\section{CONDENSING ALL THE POINTLIKE EXCITATIONS TO OBTAIN A TRIVIAL TOPOLOGICAL ORDER}

Starting in this section, we show the main result of the paper via the four steps outlined at the end of the Introduction. First, we show that condensing all the pointlike excitations in $3+1 \mathrm{D}$ always gives us a trivial topological order.

\section{A. There is no 3+1D topological order with only nontrivial stringlike excitations}

Such a result can be shown using the principle of remote detectability in Sec. III A. When there is no nontrivial pointlike excitations, the remote detectability condition requires that a single loop of string can be remotely detected by braiding other stringlike excitations around the loop. Such a braiding is the two-string braiding shown in Fig. 4(a), where a string $s_{2}$ is braided around a loop $s_{1}$.

We can also use the dimension-reduction picture to show that the anomaly-free condition requires that the two-string braiding is nontrivial. Since there are no nontrivial pointlike excitations, the dimension reduction contains only the untwisted sector. In the $2+1 \mathrm{D}$ dimension-reduced topological order, all the nontrivial anyons come from the pure strings in the $3+1 \mathrm{D}$ topological order and correspond to
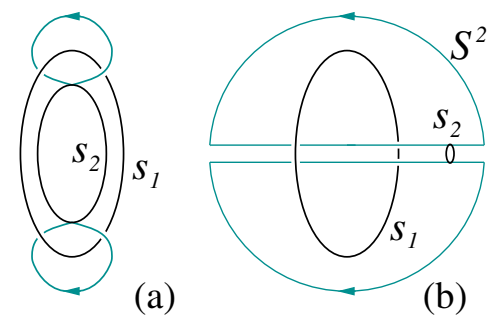

(b)

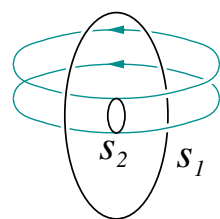

(c)
FIG. 4. The braiding path of moving the string $s_{2}$ around $s_{1}$. Panels (a)-(c) describe the same kind of braiding paths that can deform into each other smoothly.

the pure strings wrapping around the compactified $S^{1}$. The $2+1 \mathrm{D}$ topological order is anomaly-free, and the anyons form a modular tensor category. Physically, this means that any nontrivial anyon must have nontrivial mutual statistics with some anyons. This requirement implies that any nontrivial pure strings in $3+1 \mathrm{D}$ must have nontrivial two-string braiding with some strings.

Next, we show that the two-string braiding is always trivial when there is no nontrivial pointlike excitations. This is because the braiding path of an $s_{2}$ string around an $s_{1}$ string in Fig. 4(a) is a torus wrapped around loop $s_{1}$. Such a torus can be deformed into a sphere $S^{2}$ around $s_{1}$, with a thin tube going through its center [see Fig. 4(b)]. If the total space is a 3 -sphere $S^{3}$, we can deform the sphere $S^{2}$ into a small sphere on the other side of $S^{3}$. This deforms the braiding path of $s_{2}$ into a thin torus that describes a small string $s_{2}$ braiding around loop $s_{1}$ [see Fig. 4(c)]. This is like shrinking the string $s_{2}$ into a point and letting the point braid around loop $s_{1}$. Since there are no nontrivial pointlike excitations, the point that represents the small $s_{2}$ must have a trivial braiding phase around loop $s_{1}$. In this way, we show that the two strings must have trivial braiding around each other when there are no nontrivial pointlike excitations. Therefore,

the 3+1D topological orders with only stringlike excitations cannot exist (i.e., they must be anomalous).

\section{B. There are no nontrivial stringlike excitations that have trivial braiding with all pointlike excitations}

Let us assume that there is a nontrivial stringlike excitation $s$ that has trivial braiding with all pointlike excitations. If all the pointlike excitations are bosons, then we can condense all the pointlike excitations to obtain a new $3+1 \mathrm{D}$ topological order, which will have no nontrivial pointlike excitations. But since the string $s$ has trivial braiding with all pointlike excitations, it can survive the condensation and become a nontrivial stringlike excitation in the new $3+1 \mathrm{D}$ topological order.

However, in the last section, we show that $3+1 \mathrm{D}$ topological orders with only stringlike excitations cannot exist. This contradiction implies that 
there are no nontrivial stringlike excitations with trivial braiding with all pointlike excitations, if all the pointlike excitations are bosons.

This result also implies that

the untwisted sector of a dimension-reduced 3+1D topological order is a minimal modular extension of $\mathcal{R e p}(G)$.

because, in the untwisted sector, other anyons besides $\operatorname{Rep}(G)$ all come from strings in $3+1 \mathrm{D}$ and have nontrivial braiding with the particles in $\operatorname{Rep}(G)$. Thus, the modular extension is minimal. However, the above result is weaker than that obtained in Sec. III C.

\section{Condensing all the pointlike excitations gives rise to a trivial $3+1 \mathrm{D}$ topological order}

When all the pointlike excitations are bosons, we can obtain a new topological order by condensing all the pointlike excitations. The new topological order has no nontrivial pointlike excitations (since they are all condensed) or nontrivial stringlike excitations (they are confined because of the nontrivial braiding with the pointlike excitations). Thus, the new topological order must be an invertible topological order. But in $3+1 \mathrm{D}$, all the invertible topological orders are trivial ones [20,40,41]. Hence, condensing all the pointlike excitations gives us a trivial $3+1 \mathrm{D}$ topological order.

In a gauge theory, condensing all the pointlike excitations corresponds to condensing all the charged excitations, which breaks all the "gauge symmetry." This will give us an Anderson-Higgs phase, which is a trivial phase with no topological order.

\section{STRING-ONLY BOUNDARY OF 3 + 1D TOPOLOGICAL ORDER}

\section{A. The canonical gapped boundary of $3+1 D$ topological order}

In this section, we study a particular boundary of $3+1 \mathrm{D}$ topological orders. In Ref. [20], it was argued that all $3+1 \mathrm{D}$ bosonic topological orders can have a gapped boundary. The argument goes as follows.

First, topological orders have an important topological invariant: the ground-state degeneracy on closed spaces [42]. To obtain a more detailed characterization of topological orders, we consider degenerate ground states on closed spaces with different metrics. The set of closed spaces with different metrics forms the moduli space. So the degenerate ground states on closed spaces with different metrics form a vector bundle over the moduli space [7-9]. Such a vector bundle should fully characterize topological orders.

We can view the space as a boundary of the space-time. If the above vector bundle admits a flat connection, this means that the corresponding partition function on the space-time with a boundary can be independent of the perturbation of space-time metrics. This implies that for such a special realization of the topological order, the correlations of local operators on the boundary are short ranged; i.e., the boundary is gapped.

If the vector bundle does not admit a flat connection (i.e., when the Chern class is nontrivial), then the nonflat connection must be a $U(1)$ connection since the groundstate degeneracy cannot be lifted by any perturbations. Such a nonflat $U(1)$ connection is described by a gravitational Chern-Simons term in the partition function on closed space-time (for more details, see Ref. [20]). But in $3+1 \mathrm{D}$, there is no gravitational Chern-Simons term. So the vector bundle always admits a flat connection. Thus, all $3+1 \mathrm{D}$ bosonic topological orders can have a gapped boundary. We call such a gapped boundary an anomalous $2+1 \mathrm{D}$ topological order.

It is known in the theory of $2+1 \mathrm{D}$ topological orders that the interface induced by boson condensation, between the original phase and the condensed phase, is always gappable. We believe that this is also true in higher-dimensional topological orders. The idea is as follows. In the cleanest case, where we do nothing more than force the desired bosonic pointlike excitations to condense, new excitations in the condensed phase and on the interface in between should all come from the original phase. More precisely, those excitations that have nontrivial braiding with the condensed boson can no longer move freely in the condensed phase; they are confined and stuck on the interface. On the other hand, those excitations that have trivial braiding with the condensed boson survive and become excitations in the condensed phase. Since all the excitations in the original phase are gapped, we believe that both the condensed phase and the interface are also gapped. When the condensed phase is trivial, the interface is a gapped boundary.

From the last section, we see that every $3+1 \mathrm{D} A B$ topological order (where all pointlike excitations are bosons) has a boundary obtained by condensing all the pointlike excitations. Such a boundary has no topologically nontrivial pointlike excitations; it has only stringlike excitations. In light of the above argument, we believe that such a string-only boundary is gapped:

\section{Every $3+1 \mathrm{D} A B$ topological order has a string-only gapped boundary.}

\section{B. Unitary pointed fusion 2-category}

We show that the string-only gapped boundary is described by a so-called unitary pointed fusion 2-category. But what is a fusion 2-category? In general, a fusion category describes the fusion of codimension-1 excitations, i.e., domain-wall excitations. In one-dimensional space, the domain-wall excitations are pointlike. The fusion of those pointlike excitations in 1D space is described by a fusion 


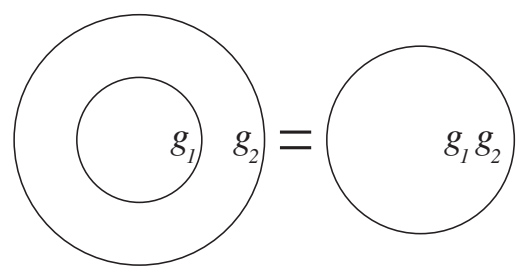

FIG. 5. The fusion of boundary stringlike excitations $s_{g_{1}}^{\text {bdry }} \otimes$ $s_{g_{2}}^{\text {bdry }}=s_{g_{1} g_{2}}^{\text {bdry }}$, which can be abbreviated as $g_{1} \otimes g_{2}=g_{1} g_{2}$.

1-category (which is also called the fusion category). In two-dimensional space, the domain-wall excitations are stringlike. The fusion of those stringlike excitations in 2D space is described by a fusion 2-category.

We point out that the fusion 2-category that describes the string-only boundary of $3+1 \mathrm{D} \mathrm{AB}$ topological order is very special: (1) The stringlike excitations on the boundary are labeled by the group elements of $G: s_{g}^{\text {bdry }}, g \in G$. (2) The fusion of the boundary stringlike excitations (see Fig. 5) is very simple and is given by the group multiplication

$$
s_{g_{1}}^{\text {bdry }} \otimes s_{g_{2}}^{\text {bdry }}=s_{g_{1} g_{2}}^{\text {bdry }} \text {. }
$$

The fusion 2-categories with the above type of fusion rule are called pointed fusion 2-categories. Such an amazing result is a consequence of condensing all the pointlike excitations described by $\mathcal{R e p}(G)$.

One way to show the above result is to consider the untwisted sector of the dimension reduction, which is a $2+$ 1D topological order. We have shown that the untwisted sector is a minimal modular extension of $\operatorname{Rep}(G)$ in Secs. IIIC and IVB. The $2+1 \mathrm{D}$ boundary with only strings corresponds to a $1+1 \mathrm{D}$ boundary with only particles of the untwisted sector in the dimension-reduced $2+1 \mathrm{D}$ topological order. Such a $1+1 \mathrm{D}$ boundary is obtained by condensing all the anyons in $\mathcal{R} \operatorname{ep}(G)$. The corresponding mathematical problem has already been solved; see, for example, Ref. [43]. We reorganized the related mathematical results and provided physical interpretations in Ref. [14] (Sec. VID). We find that the particles on such a $1+1 \mathrm{D}$ boundary of the untwisted sector are labeled by group elements in $G$ with a fusion given by group multiplication. Those $1+1 \mathrm{D}$ boundary particles correspond to the strings on the $2+1 \mathrm{D}$ boundary (see Fig. 1); this allows us to solve Eq. (20). In the next few sections, we give a different argument without using dimension reduction.

\section{Tannaka duality in more explicit language}

Our argument relies heavily on the Tannaka duality, or Tannaka reconstruction theorem for group representations. This is exactly how we extract the group $G$ from an abstract SFC. A naive example is that, for an Abelian group, the tensor product of its irreducible representations has exactly the same group structure, which can be viewed as a Fourier transformation.
In more general cases, one can reconstruct a group $G$ from its representation category $\mathcal{R e p}(G)$ by the automorphisms of a fiber functor, namely, a functor $F$ from $\mathcal{R e p}(G)$ to the category of vector spaces vect, that preserves the fusion and braiding. We know that the category of vector spaces vect describes particles in a trivial phase (i.e., in a product state with no symmetry). So one way to physically realize a fiber functor is by condensing (or in other ways such as symmetry breaking) a nontrivial phase to a trivial phase. With a fiber functor $F$, we have

$$
G \cong \operatorname{Aut}(F: \mathcal{R e p}(G) \rightarrow \text { Vect }) .
$$

To understand the physical significance of the amazing result above, let us consider a physical problem: Given a system with a symmetry whose ground state is a product state with the symmetry, if we only measure the system via probes that do not break the symmetry, can we determine the symmetry group of the system? Here, symmetric probes correspond to operators $O$ that commute with all group actions, $g O g^{-1}=O, \forall g$. Generic group actions are not symmetric probes unless they are in the center of the group. On the other hand, the fusion and braiding of the pointlike excitations above the ground state correspond to symmetric operation. The representation category $\operatorname{Rep}(G)$ contains only those symmetric probes. Tannaka duality tells us that we can indeed determine the symmetry group via only symmetric probes. Although the fiber functor seems to break the symmetry if we realize it physically, mathematically it is proven that such a fiber functor always exists and is unique up to isomorphisms. Therefore, from the data of symmetric probes (fusion and braiding) in $\mathcal{R} \operatorname{ep}(G)$, we can obtain (formally calculate) the group $G$ up to isomorphisms, without really breaking the symmetry of the system.

Now, let us try to break the abstract theorem into more explicit terms. First, the fiber functor is nothing but realizing the abstract fusion and braiding in the $\mathcal{R} \operatorname{ep}(G)$ category with the tensor product and (trivial) braiding of concrete Hilbert spaces in a quantum system. It is helpful to consider how we build $\mathcal{R e p}(G)$ in vect: A group representation is a vector space $V$ equipped with a group action $\rho_{V}: G \rightarrow \mathrm{GL}(V)$. Moreover, there is a monoidal structure for the representations, which means taking the tensor product of the vector spaces $V \otimes_{\mathbb{C}} W$, and the new group action is $\rho_{V \otimes_{\mathbb{C}} W}(g)=\rho_{V}(g) \otimes_{\mathbb{C}} \rho_{W}(g)$ (which is called the fusion of group representations).

The Tannaka duality goes exactly the other direction. Assuming that we know a representation category $\mathcal{R e p}(G)$, which contains only information on symmetric operations such as how the representations fuse with each other, can we obtain the group actions and also the group? The answer to the theorem is as follows:

(1) If we have a collection of invertible linear maps $\alpha_{X}$ for each irreducible representation $X$, acting on the vector space $F(X)$ assigned to $X$ by a fiber functor $F$, such that 
(2) They are compatible with the fusion, in the sense that $\alpha_{X \otimes Y}=\alpha_{X} \otimes_{\mathbb{C}} \alpha_{Y}$,

$$
\begin{array}{cc}
F(X \otimes Y) & \sim F(X) \otimes_{\mathbb{C}} F(Y) \\
\downarrow \alpha_{X \otimes Y} & \downarrow_{\alpha_{X} \otimes \mathbb{C} \alpha_{Y}} \\
F(X \otimes Y) & \sim F(X) \otimes_{\mathbb{C}} F(Y),
\end{array}
$$

then it is possible that $X \otimes Y$ is a reducible representation. We extend the linear maps $\alpha_{W}$ to $W$, being reducible representations by direct sums; i.e., if $W$ is the direct sum of irreducible representations $W_{i}$, $W=\bigoplus_{i} W_{i}, \alpha_{W}$ is given by the corresponding direct sum $\alpha_{W}=\bigoplus_{i} \alpha_{W_{i}}$.

This collection of invertible linear maps $\alpha_{X}$ must correspond to the action of some group element $g \in G$, $\alpha_{X}=\rho_{F(X)}(g)$.

Moreover, if we take all collections of such invertible linear maps, they form a group under composition, namely, the automorphism group of the fiber functor, $\operatorname{Aut}(F: \mathcal{R e p}(G) \rightarrow$ Vect $)$. It is isomorphic to $G$. In other words, if at the beginning we are given an abstract bosonic $\mathrm{SFC} \mathcal{E}$, with a fiber functor $F: \mathcal{E} \rightarrow$ Vect, we can use the above reconstruction to extract the group underlying $\mathcal{E}$, via $\mathcal{E} \cong \mathcal{R e p}(\operatorname{Aut}(F: \mathcal{E} \rightarrow$ Vect $))$.

\section{Fusion of boundary strings recover the group}

Let us focus on the loop excitations on the string-only boundary. A loop excitation that is shrunk to a point may become a direct sum of pointlike excitations [see Eq. (A20)],

$$
s=n \mathbf{1} \oplus \cdots,
$$

where $\mathbf{1}$ is the trivial pointlike excitation and $\cdots$ represents other possible nontrivial pointlike excitations. When $n=0$, the string is not pure. Another possibility is that $n>1$. In this case, the string is unstable; it has accidental degeneracy, which can be lifted by perturbations. So the pure simple strings have $n=1$.

Since there is only a trivial particle on the boundary, when we shrink a loop on the boundary, it must become a multiple of the trivial particle, $n \mathbf{1}$. Thus, it suffices to consider only the simple loops $(n=1)$ on the boundary, which shrink to the trivial particle 1 . In other words, simple loops on the boundary shrink to nothing; this is an essential property in the following discussions. We note that such simple loops have a quantum dimension $d=1$, and their fusion is grouplike. For the moment, we denote the group formed by the simple loops on the boundary under fusion (see Fig. 5) by $H$.

To apply the Tannaka duality, we need a physical realization of the fiber functor. Consider a simple topology for a string-only boundary: Put the $3+1 \mathrm{D}$ topological order $\mathcal{C}^{4}$ in a 3-disk $D^{3}$, the boundary on $\partial D^{3}=S^{2}$, and the

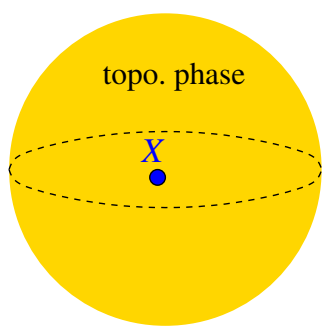

(a)

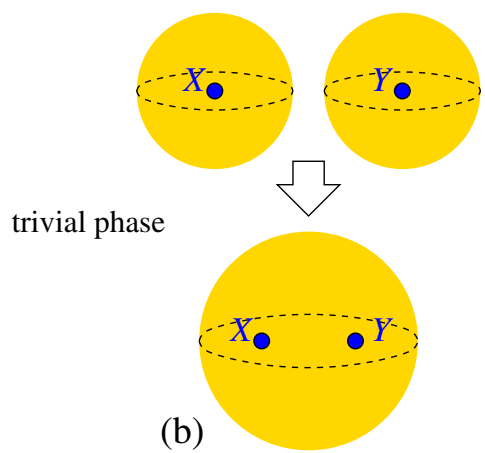

(b)
FIG. 6. (a) The fusion space $F(X)$ for a 3-disk $D^{3}$ containing only one particle $X$. (b) Merging two 3-disks to one 3-disk induces an isomorphism $F(X) \otimes_{\mathbb{C}} F(Y) \cong F(X \otimes Y)$.

trivial phase $\mathcal{D}^{4}$ outside. When there is only a particle $X$ in the 3-disk, with no string and no other particles, we associate the corresponding fusion space (the physical states with such a configuration) to the particle $X$, and we denote this fusion space by $F(X)$ (see Fig. 6). Viewed from very far away, a 3-disk containing a particle $X$ is like a "local excitation" in the trivial phase; thus, $F(X)$ mimics a local Hilbert space. When there are two 3-disks, each containing only one particle $-X$ and $Y$, respectivelythe fusion space is $F(X) \otimes_{\mathbb{C}} F(Y)$. Moreover, as adiabatically deforming the system will not change the fusion space, we can "merge" the two 3-disks to obtain one 3-disk containing one particle $X \otimes Y$. Therefore, $F(X) \otimes_{\mathbb{C}} F(Y) \cong$ $F(X \otimes Y)$. Similarly, $F$ also preserves the braiding of particles. In other words, the assignment $X \rightarrow F(X)$ gives rise to a fiber functor. By Tannaka duality, we can, at least formally, reconstruct a group $G=\operatorname{Aut}(F)$, such that the particles in the bulk $\mathcal{C}^{4}$ are identified with $\mathcal{R e p}(G)$. Our goal is to show that the fusion group $H$ of the simple loops on the boundary is the same as $G$.

To attain this goal, we consider the process of adiabatically moving a particle $X$ around a simple loop $h \in H$ on the boundary, as shown in Fig. 7. As the simple loop shrinks to nothing, inserting simple loops will not change the fusion space. But an initial state $\left|v_{0}\right\rangle \in F(X)$, after such an adiabatically moving process, can evolve into a different end state $\left|v_{1}\right\rangle \in F(X)$. Thus, braiding $X$ around $h$ induces

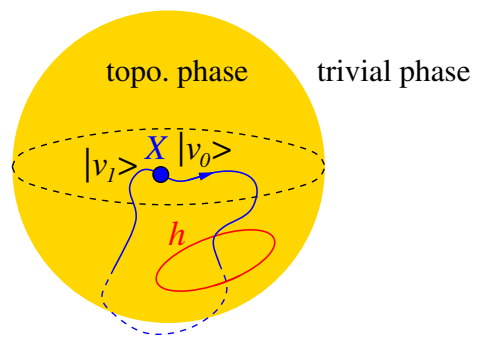

FIG. 7. Moving a particle (blue) around a loop excitation (red) on the boundary. The solid line is a half-braiding path. The dashed line is the complementary path in the trivial phase. 
an invertible (since we can always move $X$ backwards) linear map on the fusion space $F(X), \alpha_{X, h}:\left|v_{0}\right\rangle \mapsto\left|v_{1}\right\rangle$.

Next, consider that we have two particles $X, Y$ in the bulk. If we braid them together (fusing them to one particle $X \otimes Y$ ) around the simple loop $h$, we obtain the linear map $\alpha_{X \otimes Y, h}$. If the fusion of the bulk particles is given by $X \otimes Y=\bigoplus_{i} W_{i}$, we can split $X \otimes Y$ into the irreducible representations $W_{i}$, and braid $W_{i}$ with $h$; in other words, $\alpha_{X \otimes Y, h}=\bigoplus_{i} \alpha_{W_{i}, h}$.

However, this is also equivalent to moving $X$ and $Y$ one by one successively. More precisely, we can first separate $Y$ into another 3-disk, braid $X$ with $h$, and then merge $Y$ back to the original 3-disk. Thus, moving $X$ corresponds to the linear map $\alpha_{X, h} \otimes_{\mathbb{C}} i d_{F(Y)}$. Similarly, moving $Y$ corresponds to $i d_{F(X)} \otimes_{\mathbb{C}} \alpha_{Y, h}$, and in total, we have the linear map $\alpha_{X, h} \otimes_{\mathbb{C}} \alpha_{Y, h}$. Therefore, $\alpha_{X \otimes Y, h}=\alpha_{X, h} \otimes_{\mathbb{C}} \alpha_{Y, h}$, or using only irreducible representations,

$$
\alpha_{X, h} \otimes_{\mathbb{C}} \alpha_{Y, h}=\bigoplus_{i} \alpha_{W_{i}, h}
$$

These linear maps are compatible with the fusion of bulk particles.

Moreover, the simple loop $h$ provides such an invertible linear map $\alpha_{X, h}$ for each particle $X \in \mathcal{R} \operatorname{ep}(G)$ in $\mathcal{C}^{4}$; thus, by Tannaka duality, these linear maps must correspond to the action of certain group elements $\varphi(h) \in G$, $\alpha_{X, h}=\rho_{F(X)}(\varphi(h))$. In other words, we obtain a map $\varphi$ from the simple loops $H$ to $G, \varphi: H \rightarrow G$. It is compatible with the fusion of simple loops because the path of braiding around two concentric simple loops $g_{1}, g_{2}$ (as in Fig. 5), separately, can be continuously deformed to the braiding path around the two loops together, or around their fusion $g_{1} \otimes g_{2}=g_{1} g_{2}$. This implies that $\varphi\left(g_{1}\right) \varphi\left(g_{2}\right)=\varphi\left(g_{1} g_{2}\right)$; namely, $\varphi$ is a group homomorphism.

We want $\varphi$ to be an isomorphism and $H=G$. This is a consequence of the remote detectability condition. Before proving it, we explain in detail the principle of remote detectability near the string-only boundary. The general idea is the same, that everything must be detectable remotely. Near a string-only boundary, the only way to perform remote detection is by the half-braiding between bulk particles and boundary strings $[20,36]$. Therefore,

(1) there is no nontrivial boundary string that has trivial half-braiding with all the bulk particles (boundary strings are detectable by bulk particles), and (2) there is no nontrivial bulk particle that has trivial halfbraiding with all the boundary string.

One may have doubts about point (2): Even if bulk particles cannot be detected by boundary strings, we may still have bulk strings to detect them. The reason for (2) is that we believe generalized boundary-bulk duality-that the bulk strings can always be viewed as a certain "lift" of boundary strings to the bulk $[20,21]$. If a bulk particle has trivial half-braiding with all boundary strings, it also has trivial braiding with all the "lift" of boundary strings, i.e., all the bulk strings, which conflicts with the remote detectability condition in the bulk.

A typical half-braiding path is shown in Fig. 7. It is important to note that the (non-Abelian) geometric phase depends on the half-braiding path; however, we can extract a universal path-independent half-braiding invariant by complementing the half-braiding path into a full loop with another half loop of path in the trivial phase outside the boundary. Different half loops of the path in the trivial phase with the same starting and end points on the boundary always contribute the same geometric phase (because closed paths in the trivial phase have no geometric phase). Thus, we obtain the half-braiding invariant as the expectation value of such a whole-loop, adiabatically moving process (half in the bulk, half in the trivial phase) [44]. Trivial half-braiding means that such a half-braiding invariant is trivial. Immediately, we see that the linear maps $\alpha_{X, h}$ are directly related to the half-braidings, in the sense that $\left\langle\alpha_{X, h}\right\rangle$ gives the above half-braiding invariant. If $\alpha_{X, h}$ is the identity map, it implies trivial half-braiding between $X$ and $h$.

Now, we are ready to show that $\varphi: H \rightarrow G$ is an isomorphism:

(1) $\varphi$ is injective. Consider $\operatorname{ker} \varphi$, namely, the simple loops that induce only identity linear maps on all bulk particles. In other words, $\operatorname{ker} \varphi$ consists of simple loops that have trivial half-braiding with all bulk particles. By the remote detectability condition (1), $\operatorname{ker} \varphi$ must be trivial, which means $\varphi$ is injective.

(2) $\varphi$ is surjective. We have already shown that $\varphi$ : $H \rightarrow G$ is injective, so we can view $H$ as a subgroup of $G$.

Now, consider a special particle in the bulk, which carries the representation $\operatorname{Fun}(G / H)$, linear functions on the right cosets $G / H$. More precisely, $\operatorname{Fun}(G / H)$ consists of all linear functions on $G$, $f: G \rightarrow \mathbb{C}$, such that $f(h x)=f(x), \forall h \in H, x \in G$ (takes the same value on a coset). The group action is the usual one on functions, $\rho_{\text {Fun }(G / H)}(g): f(x) \mapsto$ $f\left(g^{-1} x\right)$.

The linear maps $\alpha_{X, h}$ induced by the simple loops are all actions of group elements in $H$, and they are all identity maps on the special particle $\operatorname{Fun}(G / H)$. In other words, the bulk particle $\operatorname{Fun}(G / H)$ has trivial half-braiding with all the boundary strings. By the remote detectability condition (2), it must be trivial particle carrying the trivial representation. In other words, we have $G=H$.

To conclude, the simple loop excitations on the stringonly boundary form a group under fusion. It is exactly the same group whose representations are carried by the pointlike excitations in the bulk. 
If we insert a bulk loop excitation in the 3-disk and perform a similar braiding process, it also induces a linear map on the underlining fusion space. One may wonder if this also associates group elements to the bulk strings. This is, in general, not true. Unlike the boundary simple loops, inserting a bulk string, even if it is pure and simple, will enlarge the fusion space of only particles, as long as the quantum dimension of such a string is greater than 1 . As a result, only those bulk strings with quantum dimension $d=1$ can be associated with group elements. In Sec. III C, we have shown that all the bulk strings can be associated with conjugacy classes of group $G$ (even if topological order is not a $G$-gauge theory). Some further discussions can be found in Appendix C.

\section{CLASSIFICATION OF UNITARY POINTED FUSION 2-CATEGORIES}

\section{A. Mathematical formulation}

First, let us consider the so-called unitary pointed fusion (1-)categories. A pointed fusion category consists of a finite number of simple objects. A simple object $x$ is an object such that $\operatorname{Hom}(x, x)=\mathbb{C}$. For each simple object $x$, there is a simple object $y$ such that $x \otimes y=1$, where 1 is the tensor unit, i.e., $1 \otimes x=x=x \otimes 1$. In other words, the set of simple objects form a finite group $G$. We also denote the simple object by $g_{1}, g_{2}, g_{3}$, etc.

In this case, the only not-yet-fixed structure is the associator isomorphism:

$$
\left(g_{1} \otimes g_{2}\right) \otimes g_{3} \rightarrow g_{1} \otimes\left(g_{2} \otimes g_{3}\right) .
$$

Note that both the domain and the target are the same simple object. But the associator isomorphisms can be nontrivial. By the simpleness, the isomorphism is just a nonzero complex number $\omega_{3}\left(g_{1}, g_{2}, g_{3}\right)$. If the theory is unitary, one needs to further require that this number is a phase [in $U(1)]$. Then, the pentagon condition implies that $\omega_{3}\left(g_{1}, g_{2}, g_{3}\right)$ is a 3-cocycle. Different cocycles give equivalent fusion categories if they differ by a 3 -coboundary. Moreover, we may permute the simple objects by group automorphisms; thus, two different cocycles also give equivalent fusion categories if they get mapped to each other by group automorphisms. In conclusion, unitary pointed fusion categories are classified by $\left(G, \omega_{3}\right)$, where $\omega_{3} \in \mathcal{H}^{3}[G ; U(1)]$ up to group automorphisms.

Now, let us consider a pointed unitary fusion 2-category [45]. We will not define it in full detail here but only describe some of its physically relevant ingredients. It has only a finite number of simple objects. A generic object is a direct sum of simple objects. For two simple objects $x, y$, we have $\operatorname{Hom}(x, x)=\operatorname{Vect}$ and $\operatorname{Hom}(x, y)=0$ for $x \neq y$, where 0 is the category consisting of only the 0 -vector space. The tensor unit 1 is simple. For each simple object $x$, there is a simple object $y$ such that $x \otimes y=1$, where 1 is the tensor unit. So, again, the set of simple objects is a finite group $G$. We denote simple objects by group elements $g_{1}$, $g_{2}, g_{3}$, etc.

For a simple object $g$, the identity 1-morphism id is $\mathbb{C}$ (the only invertible object in Vect). The 2-morphisms form $\operatorname{Hom}\left(\mathrm{id}_{g}, \operatorname{id}_{g}\right)=\operatorname{Hom}_{\text {Vect }}(\mathbb{C}, \mathbb{C})=\mathbb{C}$, and there are unit 1isomorphisms and associator 1-isomorphisms: (1) The unit 1-isomorphism $1 \otimes g=g \rightarrow g$ is just the identity morphism $i d_{g}=\mathbb{C}$. (2) The associator 1-isomorphism

$$
\left(g_{1} \otimes g_{2}\right) \otimes g_{3} \rightarrow g_{1} \otimes\left(g_{2} \otimes g_{3}\right)
$$

is also the identity 1-morphism $i d_{g_{1} g_{2} g_{3}}=\mathbb{C}$. There are two ways to go from $\left(\left(g_{1} g_{2}\right) g_{3}\right) g_{4}$ to $g_{1}\left(g_{2}\left(g_{3} g_{4}\right)\right)$ via identity 1-morphisms. Therefore, the two paths give the identity map $\operatorname{id}_{g_{1} g_{2} g_{3} g_{4}}=\mathbb{C}$. So the commutativity of the pentagon diagram is clear. However, we can introduce, for each pentagon, a 2-isomorphism $\mathbb{C} \rightarrow \mathbb{C}$, which is a phase denoted by $\omega_{4}\left(g_{1}, g_{2}, g_{3}, g_{4}\right)$. This 2 -isomorphism needs to satisfy a higher coherence relation. Then, this coherence relation implies that $\omega_{4}\left(g_{1}, g_{2}, g_{3}, g_{4}\right)$ is a 4-cocycle. Again, 4-cocycles differing only by a coboundary give equivalent pointed fusion 2-categories. One can do the same for the triangle relation. Namely, one can introduce a 2-isomorphism for each triangle. We believe that these 2-isomorphisms should give the same unitary fusion 2-category.

The same structure is discussed in Ref. [46], under a different name, $G$-graded 2-vector spaces $2 \mathbf{V e c}_{G}^{\omega_{4}}$, where they also believe that $\left(G, \omega_{4}\right)$ is enough to determine a unitary pointed fusion 2-category.

Note that the equivalence between unitary pointed fusion 2-categories must preserve the tensor product of simple objects; thus, it must correspond to some group automorphism $\phi: G \cong G$. Such an automorphism also acts on the cocycles (it necessarily changes the cocycle if it is an outer automorphism, namely, not of the form $x \mapsto g x g^{-1}$ for some $g \in G)$. Under such automorphism $\phi,\left(G, \omega_{4}\right)$ and $\left(G, \phi\left(\omega_{4}\right)\right)$, where $\omega_{4}$ is a 4-cocycle, correspond to the same pointed unitary fusion 2-category. Therefore, we believe that pointed unitary fusion 2-categories correspond one-to-one to the pairs $\left(G, \omega_{4}\right)$, where $\omega_{4} \in \mathcal{H}^{4}[G ; U(1)]$, up to group automorphisms.

\section{B. Physical argument}

In the following, we try to understand the above mathematical result from a physical point of view. Let the three-dimensional space be a 3-disk $D^{3}$. Consider the boundary strings $s_{g_{1}}^{\text {bdry }}$ and $s_{g_{2}}^{\text {bdry }}$ on the surface of the 3-disk $S^{2}=\partial D^{3}$ [see Fig. 8(a)]. The process for the boundary strings to fuse to a no-string state can be represented by a membrane net in $D^{3}$ (see also Ref. [46]). The same boundary strings can fuse to a no-string state through a 

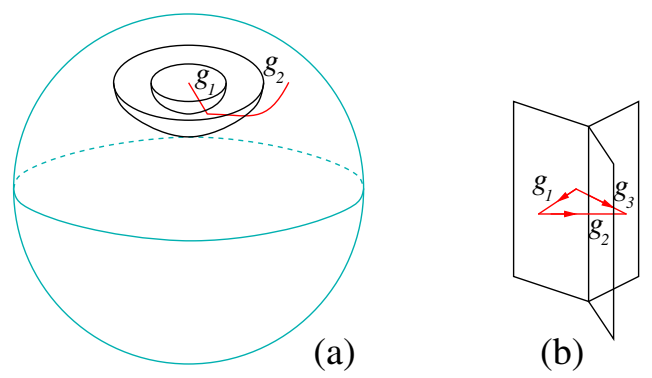

(b)

FIG. 8. (a) Two boundary strings $s_{g_{1}}^{\text {bdry }}$ and $s_{g_{2}}^{\text {bdry }}$ on the surface $S^{2}$ of $D^{3}$. The process of fusing the two strings to a no-string state is described by a membrane net in $D^{3}$ (which is formed by two hemispheres, in this case). The dual of the membrane net is a string net (which is formed by two strings, in this case). (b) The intersection of three membranes is dual to a triangle of strings. The fusion rule of the boundary strings corresponds to the flatconnection condition Eq. (28) on the string net.

different process, which is represented by another membrane net in $D^{3}$. To compare the two processes, we can glue the boundary of the above two membranes together along $S^{2}$ to form a membrane net in $S^{3}$. Such a membrane net in $S^{3}$ describes the process of creating boundary strings from a no-string state and then fusing those boundary strings to a no-string state. This process induces a $U(1)$ geometric phase $\mathrm{e}^{\mathrm{i} \theta}$ since the fusion space of the boundary strings is always one dimensional. Thus, we assign such a $U(1)$ geometric phase $\mathrm{e}^{\mathrm{i} \theta}$ to the membrane net on $S^{3}$.

But such a $U(1)$ geometric phase may not have a local expression. Let us assume that the membrane net on $S^{3}$ is formed by the 2-simplices of a triangulation of $S^{3}$. The vertices of the triangulation are labeled by $I, J, K, \ldots$. "Having no local expression" means that we cannot assign a phase factor $\omega_{3}(I J K L)$ to each 3-simplex $\langle I J K L\rangle$ of the triangulation to express the total $U(1)$ geometric phase $\mathrm{e}^{\mathrm{i} \theta}$ as a product of those local phases:

$$
\mathrm{e}^{\mathrm{i} \theta} \neq \prod_{\langle I J K L\rangle} \omega_{3}(I J K L) .
$$

We see that the process of creating some boundary strings from nothing and then fusing them to nothing can be represented by a membrane net on space $S^{3}$. Such a process corresponds to a phase factor $\mathrm{e}^{\mathrm{i} \theta}$. Two different processes of creating some boundary strings from nothing and then fusing them to nothing give rise to two phase factors $\mathrm{e}^{\mathrm{i} \theta}$ and $\mathrm{e}^{\mathrm{i} \theta^{\prime}}$. The two processes can be compared by a "time" evolution from the membrane net on $S^{3}$ that corresponds to the first process to the membrane net on $S^{3}$ that corresponds to the second process. In other words, the comparison of the two processes is represented by a 3-brane net on $S^{3} \times I$, where $S^{3}$ is the space and the segment $I$ represents the time direction (see Fig. 9). The first process corresponds to the membrane net on one boundary of $S^{3} \times I$, which is one boundary of the 3-brane net on $S^{3} \times I$. The second process

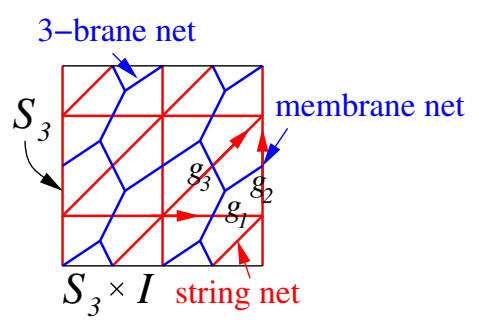

FIG. 9. Two processes are described by the membrane net on the two boundaries of $S^{3} \times I$. The change between the two processes is described by a 3-brane net (blue lines) on $S^{3} \times I$ (with two of the three dimensions suppressed). The red lines form the string net, which is dual to the 3-brane net.

corresponds to the membrane net on the other boundary of $S^{3} \times I$, which is the other boundary of the 3-brane net on $S^{3} \times I$.

In four dimensions, a 3-brane net is dual to a string net where each 3-brane in the 3-brane net intersects with a string in the string net (see Figs. 8 and 9). Thus, the strings in the string net are also labeled by $g_{i}$. In the 3-brane net, only the 3-branes that satisfy the fusion rule Eq. (20) can intersect along a line [see Figs. 8(b) and 9]. This means that the labels of the strings in the string net satisfy

$$
g_{1} g_{2}=g_{3} .
$$

[Note the same string with opposite orientations is labeled by $g$ and $g^{-1}$, respectively. The orientation of strings in the string net is chosen to form a branching structure (see Appendix E) of the string net.] The above happens to be the flat-connection condition if we view $g_{i}$ on a string as the gauge connection between the two vertices connected by the string. Thus, the evolution from one process to the other can be represented by a string net on $S^{3} \times I$.

The two different processes may differ by a phase factor $\mathrm{e}^{\mathrm{i}\left(\theta^{\prime}-\theta\right)}$. Therefore, we can assign the string net on $S^{3} \times I$ by such a phase factor to represent the difference of the two processes. Let us assume the string net on $S^{3} \times I$ is formed by the edges of a triangulation of $S^{3} \times I$. Then, starting from one boundary of $S^{3} \times I$, we can build the whole triangulation of $S^{3} \times I$ by adding one pentachoron (i.e., one 4-simplex) at a time. We note that adding a pentachoron corresponds to changing one process to its neighboring process. The difference of the two neighboring processes is described by the added pentachoron with edges labeled by $g_{I J}, I, J=0,1,2,3,4$ (where $I=0,1,2,3,4$ label the five vertices of the pentachoron). We may assign the phase difference of the two neighboring processes to the added pentachoron. Thus, each pentachoron is assigned to a phase factor $\omega_{4}\left(\left\{g_{I J}\right\}\right)$. Because of the flat-connection condition Eq. (28), $g_{I J}$ 's for the pentachoron are not independent. Therefore, $\omega_{4}\left(\left\{g_{I J}\right\}\right)$ can be rewritten as $\omega_{4}\left(g_{01}, g_{12}, g_{23}, g_{34}\right)$. Such a 4 -variable function on $G$ can be viewed as a group 4-cochain. 
Thus, the total phase difference of the two processes can be written as

$$
\mathrm{e}^{\mathrm{i}\left(\theta^{\prime}-\theta\right)}=\prod_{\langle I J K L M\rangle \in S^{3} \times I} \omega_{4}^{S_{I J K L M}}\left(g_{I J}, g_{J K}, g_{K L}, g_{L M}\right),
$$

where $\prod_{\langle I J K L M\rangle \in S^{3} \times I}$ are multiplied over all the pentachorons $\langle I J K L M\rangle$ in the triangulation of $S^{3} \times I$, and $s_{I J K L M}=$ \pm 1 describes the two different orientations of the pentachorons $\langle I J K L M\rangle$, which arise from the branching structure (see Appendix E).

If we choose the two processes described by the boundary of $S^{3} \times I$ to be the do-nothing process that leaves the no-string state unchanged, then the string net on $S^{3} \times I$ can be viewed as a string net on $S^{4}$. The total phase difference of the two do-nothing processes (which are actually the same process) should be zero:

$$
1=\prod_{\langle I J K L M\rangle \in S^{4}} \omega_{4}^{s_{I J K L M}}\left(g_{I J}, g_{J K}, g_{K L}, g_{L M}\right),
$$

and the above should hold for any triangulation of $S^{4}$ and any assignment of the label $g_{I J}$ on the edges [as long as the flat-connection condition Eq. (28) is satisfied]. This result implies that $\omega_{4}\left(g_{01}, g_{12}, g_{23}, g_{34}\right)$ is a group 4-cocycle in $\mathcal{H}^{4}(G ; U(1))$. It is a physical way to explain why

unitary pointed fusion 2-categories are classified by a pair $\left(G, \omega_{4}\right)$ up to group automorphisms, where $G$ is a finite group and $\omega_{4}$ its group 4-cohomology class: $\omega_{4} \in \mathcal{H}^{4}(G ; U(1))$.

\section{FROM BOUNDARY TO BULK}

We have shown that all $3+1 \mathrm{D}$ AB bosonic topological orders can have a boundary described by pointed unitary fusion 2-category $\mathcal{M}^{3}$ whose fusion is given by group $G$. It is believed that the boundary anomalous topological order completely determines the bulk topological order [20,21]. More precisely, the bulk topological order should be given by the center $Z\left(\mathcal{M}^{3}\right)$, which can be explicitly defined by the 2-category $\operatorname{Fun}_{\mathcal{M}^{3} \mid \mathcal{M}^{3}}\left(\mathcal{M}^{3}, \mathcal{M}^{3}\right)$ of $\mathcal{M}^{3}$ - $\mathcal{M}^{3}$-bimodule 2-functors.

However, their relation can be many-to-one: Several different anomalous boundary topological orders may correspond to the same bulk topological order; in other words, the same bulk topological order can have several different gapped boundaries. Since 3+1D topological orders always have gapped boundaries, all $3+1 \mathrm{D}$ topological orders are determined by some anomalous $2+1 \mathrm{D}$ boundary topological orders. Mathematically, we say that there is a surjective map from the set of anomalous $2+1 \mathrm{D}$ boundary topological orders to the set of $3+1 \mathrm{D}$ topological orders:
$2+1 \mathrm{D}$ boundary anomalous topological orders

$\rightarrow 3+1 \mathrm{D}$ topological orders.

Furthermore, since all $3+1 \mathrm{D} \mathrm{AB}$ topological orders have a string-only boundary described by unitary pointed fusion 2-categories, we further have

$$
\begin{aligned}
& \text { unitary pointed fusion 2-categories } \\
& \qquad \rightarrow 3+1 \mathrm{D} \text { AB topological orders. }
\end{aligned}
$$

In this paper, the string-only boundary is obtained by condensing all the pointlike particles that form $\operatorname{Rep}(G)$. A natural question is whether such a condensation process, and also the resulting boundary, is unique or not.

First, we believe that the condensation of particles in $3+1 \mathrm{D}$ follows the same rule as that for condensation of anyons in $2+1 \mathrm{D}$ (at least if we restrict the $3+1 \mathrm{D}$ condensation to a $2+1 \mathrm{D}$ submanifold; see Fig. 2). Anyon condensation in $2+1 \mathrm{D}$ has been thoroughly studied. It is fully controlled by the so-called condensable algebra [47] in the category of anyons. In other words, the condensable algebra completely determines the condensed phase and the domain wall or boundary between the old phase and the condensed phase.

Thus, we should focus on the condensable algebras in $\mathcal{R e p}(G)$. They are already classified in Theorem 2.2 in Ref. [48] (see also Theorem 3.7 in Ref. [49]). There is a unique condensable algebra that condenses all the particles in $\mathcal{R e p}(G)$. It is given by $\operatorname{Fun}(G)$, the algebra of all functions on $G$. Therefore, there is only one way to condense all particles in $\operatorname{Rep}(G)$. We obtain a unique condensed phase, which is trivial. As a result, there is a unique, also canonical, string-only boundary.

Thus, we obtain an even stronger result. Each $3+1 \mathrm{D}$ $\mathrm{AB}$ topological order only has a unique boundary that corresponds to the condensation of all pointlike excitations. In other words, each $3+1 \mathrm{D}$ topological order corresponds to a unique unitary pointed fusion 2-category.

Unitary pointed fusion 2-categories classify all 3+1D $A B$ topological orders in a one-to-one fashion.

Such a result is similar to a result in one lower dimension:

Unitary fusion categories describe all 2 $+1 \mathrm{D}$ topological orders with gappable boundaries (but in a many-toone way) [50,51].

Let us briefly explain why the approach used in this paper for $3+1 \mathrm{D}$ topological orders does not apply in $2+1 \mathrm{D}$, which gives one an idea as to why we can obtain a stronger result in $3+1 \mathrm{D}$. In $3+1 \mathrm{D}$, all pointlike excitations have trivial statistics; if they are all bosons, it is a natural and canonical choice to condense all of them, and we obtain a unique string-only $2+1 \mathrm{D}$ gapped boundary. In $2+1 \mathrm{D}$, there are only pointlike excitations with nontrivial 
statistics between them. One can choose a subset of quasiparticles to condense; if the subset is big enough, one can also obtain a gapped $1+1 \mathrm{D}$ boundary. However, in general, there are several such subsets to condense, none of which is special. As a result, there is no canonical gapped $1+1 \mathrm{D}$ boundary. This essential difference makes the classification of topological orders in $3+1 \mathrm{D}$ simpler than those in $2+1 \mathrm{D}$.

\section{REALIZATION BY DIJKGRAAF-WITTEN MODELS}

Combining the results from the last few sections, we find that

$3+1 \mathrm{D}$ AB topological orders are classified by a finite group $G$ and its group 4-cocycle $\omega_{4} \in \mathcal{H}^{4}(G ; U(1))$, up to group automorphisms.

A finite group $G$ and its group 4-cocycle are the data needed to construct the Dijkgraaf-Witten model. In fact, all the $3+1 \mathrm{D} A B$ topological orders can be realized by Dijkgraaf-Witten models.

We note that 3+1D Dijkgraaf-Witten models [34] are defined on a four-dimensional simplicial complex with branching structure (see Appendix E). Let us use $I, J, \ldots$ to label the vertices of the complex. The degrees of freedoms live on the links of the complex, which are labeled by $g_{I J} \in G$, where $G$ is a finite group. Here, $g_{I J}$ satisfies a flat-connection condition

$$
g_{I J} g_{J K}=g_{I K},
$$

for any triangles $\langle I J K\rangle$. The Dijkgraaf-Witten models are defined via a path integral

$$
Z=\sum_{\left\{g_{I J}\right\}} \prod_{\langle I J K L M\rangle} \omega_{4}^{s_{I J K L M}}\left(g_{I J}, g_{J K}, g_{K L}, g_{L M}\right),
$$

where $\prod_{\langle I J K L M\rangle}$ multiply over all the 4-cells $\langle I J K L M\rangle$ whose vertices are ordered as $I<J<K<L<M$. Also, $s_{I J K L M}= \pm 1$ describes the orientation of the 4-cell $\langle I J K L M\rangle$ (see Appendix E), and $\omega_{4}$ is a group 4-cocycle $\omega_{4} \in \mathcal{H}^{4}[G ; U(1)]$.

When the space-time has a boundary, we can obtain an exactly soluble boundary by setting $g_{I J}=1$ on all the links $\langle I J\rangle$ on the boundary. Such an exactly soluble boundary is actually the string-only boundary discussed in this paper. The world-lines of topological pointlike excitations are described by Wilson lines in the bulk,

$$
\prod_{\langle I J\rangle} R\left(g_{I J}\right)
$$

where $R$ is a representation of $G$. However, on the boundary, $g_{I J}=1$ and $R\left(g_{I J}=1\right)$ is an identity matrix. All the different topological pointlike excitations become the same trivial excitation on the boundary. However, there are nontrivial stringlike excitations on the boundary. The world sheet of those boundary stringlike excitations is given by the following: Draw a membrane on the three-dimensional boundary of space-time. Change $g_{I J}$ on the links that intersect the membrane from $g_{I J}=1$ to $g_{I J}=h$. Such a change still satisfies the flat-connection condition. We see that different boundary strings are labeled by the group elements, and their fusion is given by the group multiplication. Therefore, Dijkgraaf-Witten models can realize all unitary pointed fusion 2-categories on the boundary. Using the boundary-bulk relation [20,21], we can show that Dijkgraaf-Witten models can realize all $3+1 \mathrm{D}$ AB topological orders.

\section{RELATION TO 3+1D BOSONIC SPT ORDERS}

There are two kinds of SPT orders when the symmetry group is unitary and finite: the ones whose boundaries have a pure gauge anomaly will be called pure SPT orders $[15,16]$, and the ones whose boundaries have a mixed gauge-gravity anomaly will be called mixed SPT orders [52]. In 3 + 1D space-time, the pure SPT orders are classified by group cohomology $\mathcal{H}^{4}[G ; U(1)]$, while all the mixed SPT orders are described by some elements in [52]

$$
\begin{aligned}
& \mathcal{H}^{1}\left(G ; \mathcal{H}^{3}[S O(\infty) ; U(1)]\right) \oplus \mathcal{H}^{2}\left(G ; \mathcal{H}^{2}[S O(\infty) ; U(1)]\right) \\
& \quad=\mathcal{H}^{1}(G ; \mathbb{Z}) \oplus \mathcal{H}^{2}\left(G ; \mathbb{Z}_{2}\right)=\mathcal{H}^{2}\left(G ; \mathbb{Z}_{2}\right) .
\end{aligned}
$$

For many groups, $\mathcal{H}^{2}\left(G ; \mathbb{Z}_{2}\right) \neq 0$. However, a nonzero $\mathcal{H}^{2}\left(G ; \mathbb{Z}_{2}\right)$ does not imply the existence of mixed SPT since not all the elements in $\mathcal{H}^{2}\left(G ; \mathbb{Z}_{2}\right)$ correspond to existing SPT orders.

Since $3+1 \mathrm{D}$ AB topological orders can be obtained by gauging [17] the symmetry of $3+1 \mathrm{D}$ bosonic SPT states, and since Dijkgraaf-Witten models only correspond to gauging the pure SPT states, we see that the classification results in this paper imply the following:

In $3+1 \mathrm{D}$, there is no mixed bosonic SPT order for unitary finite symmetry group $G$.

In fact, using a SPT invariant, we can directly show that for unitary finite symmetry group $G$, there is no mixed SPT orders in $3+1 D$. (However, if $G$ contains time reversal, there are mixed SPT orders in $3+1 \mathrm{D}$ [40,53].) To obtain a SPT invariant, we gauge the symmetry and put a flatconnection $A$ on a closed orientable space-time $M^{4}$. The partition function of the system on $M^{4}$ with a fixed flatconnection $A$ is the so-called SPT invariant [54-56]. If there is a mixed $3+1 \mathrm{D}$ SPT order described by $\mathcal{H}^{2}\left(G ; \mathbb{Z}_{2}\right)=\bigoplus \mathbb{Z}_{2}$, its SPT invariant will have the form

$$
Z\left(M^{4}, A\right)=\mathrm{e}^{\mathrm{i} \pi \int_{M^{4}} \omega_{1}(A) \smile \mathrm{w}_{3}+\omega_{2}(A) \smile \mathrm{w}_{2}},
$$


where $\omega_{n}(A), \mathrm{w}_{n}$ are topological $n$-cocycles in $H^{n}\left(M^{4} ; \mathbb{Z}_{2}\right)$, and $\mathrm{w}_{n}$ is also the $n$th Stiefel-Whitney class. There are many relations between Stiefel-Whitney classes and cocycles $\omega_{n}(A)$. For example, by calculating $S q^{1}\left(\omega_{1}(A) \smile \mathrm{w}_{2}\right)$ in two different ways, we find that on orientable $M^{4}$, $\omega_{1}(A) \smile \mathrm{w}_{3}=\omega_{1}(A) \smile \omega_{1}(A) \smile \mathrm{w}_{2}$ [52,57]. (Here, $S q^{n}$ is the Steenrod operation.) Thus,

$$
Z\left(M^{4}, A\right)=\mathrm{e}^{\mathrm{i} \pi \int_{M^{4}}\left[\omega_{2}(A)+\omega_{1}(A) \smile \omega_{1}(A)\right] \smile \mathrm{w}_{2}} .
$$

Similarly, $\quad\left[\omega_{2}(A)+\omega_{1}(A) \smile \omega_{1}(A)\right] \smile \mathrm{w}_{2}=S q^{2}\left[\omega_{2}(A)+\right.$ $\left.\omega_{1}(A) \smile \omega_{1}(A)\right]=\left[\omega_{2}(A)+\omega_{1}(A) \smile \omega_{1}(A)\right] \smile\left[\omega_{2}(A)+\right.$ $\left.\omega_{1}(A) \smile \omega_{1}(A)\right]$. Thus,

$$
Z\left(M^{4}, A\right)=\mathrm{e}^{\mathrm{i} \pi \int\left[\omega_{2}(A)+\omega_{1}(A) \smile \omega_{1}(A)\right] \smile\left[\omega_{2}(A)+\omega_{1}(A) \smile \omega_{1}(A)\right]} .
$$

We see that the SPT order described by the above SPT invariant is actually a pure SPT order described by $\mathcal{H}^{4}[G ; U(1)][40,52]$; hence, there is no mixed SPT order in $3+1 \mathrm{D}$ for the unitary finite symmetry group. This result supports our classification of $3+1 \mathrm{D}$ AB topological orders in terms of Dijkgraaf-Witten models. In 4+1D, there is a mixed bosonic $Z_{2}$ SPT state [52]. Gauging such a mixed $Z_{2}$ SPT state will produce a $4+1 \mathrm{D}$ AB topological order that is beyond Dijkgraaf-Witten theory.

\section{WALKER-WANG MODELS AND PARTICLE-ONLY BOUNDARIES}

We remark that Walker-Wang models [58-61] provide another quite systematic way to construct $3+1 \mathrm{D}$ bosonic topological orders. In fact, Walker-Wang models realize all $3+1 \mathrm{D}$ bosonic topological orders that have a particleonly boundaries which are described by premodular tensor categories. Such a particle-only boundary can exist for a $3+1 \mathrm{D}$ topological order, if condensing the maximum set of strings that have trivial mutual braiding will change the $3+1 \mathrm{D}$ topological order to a trivial phase.

It is known that Walker-Wang models (and the related $3+1 \mathrm{D}$ string-net models [50]) can realize $3+1 \mathrm{D}$ bosonic topological orders with emergent fermionic pointlike excitations [59]. It appears that Walker-Wang models cannot realize all 3+1D Dijkgraaf-Witten models (i.e., not all $3+1 \mathrm{D}$ bosonic topological orders with pointlike excitations are bosons).

\section{SUMMARY}

We note that $3+1 \mathrm{D}$ topological orders contain both pointlike and stringlike excitations. At first, it appears that $3+1 \mathrm{D}$ topological orders, with all the fusion and braiding of the pointlike and stringlike excitations, have a very complicated structure, which may be hard to classify. However, in this paper, we obtain a very simple classification of $3+1 \mathrm{D}$ topological orders for bosonic systems, when all the pointlike excitations are bosons: They are classified by unitary pointed fusion 2-categories, which in turn are classified by pairs $\left(G, \omega_{4}\right)$ up to group automorphisms. This gives us hope that the $3+1 \mathrm{D}$ topological orders may not be so complicated. We could obtain a simple classification even for the general case when some pointlike excitations are emergent fermions. We hope that the arguments developed in this paper are helpful for such a task, which we plan to carry out in a forthcoming work.

\section{ACKNOWLEDGMENTS}

We thank Meng Cheng, Zhenghan Wang, and Edward Witten for helpful discussions. X. G. W. is supported by NSF Grants No. DMR-1506475 and No. NSFC 11274192. Research at the Perimeter Institute is supported by the Government of Canada through Industry Canada and by the Province of Ontario through the Ministry of Research. L. $\mathrm{K}$. is supported by the start fund from Tsinghua University.

\section{APPENDIX A: EXAMPLE: 3 + 1D G-GAUGE THEORY}

To gain an intuitive understanding of $3+1 \mathrm{D}$ topological orders and to introduce the related concepts, let us study an exactly soluble local bosonic model whose ground state has a topological order described by a $3+1 \mathrm{D}$ gauge theory of a finite group $G$. Our lattice bosonic model is defined on a 3D spatial lattice whose sites are labeled by $I$. The degrees of freedom live on the links labeled by $I J$. On an oriented link $I J$, such degrees of freedom are labeled by $g_{I J} \in G$. Note that $g_{I J}$ 's on links with opposite orientations satisfy

$$
g_{I J}=g_{J I}^{-1}
$$

The Hamiltonian of the exactly soluble model is expressed in terms of string operators and membrane operators.

\section{String operators}

The string operators are labeled by $i$ 's, the irreducible representations $R_{i}\left(g_{I J}\right)$ of the gauge group $G$ [where $R_{i}\left(g_{I J}\right)$ is the matrix of the irreducible representation]:

$$
\begin{aligned}
B_{i}\left|\left\{g_{I J}\right\}\right\rangle & =\left[\operatorname{Tr} \prod_{I J \in \text { string }} R_{i}\left(g_{I J}\right)\right]\left|\left\{g_{I J}\right\}\right\rangle \\
& =\left[\operatorname{Tr} R_{i}\left(\prod_{I J \in \text { string }} g_{I J}\right)\right]\left|\left\{g_{I J}\right\}\right\rangle .
\end{aligned}
$$

We note that

$$
B_{i} B_{j}=\operatorname{Tr} \prod_{I \in \text { string }} R_{i}\left(g_{I J}\right) \otimes_{\mathbb{C}} R_{j}\left(g_{I J}\right) .
$$


We use $\otimes_{\mathbb{C}}$ to denote the usual tensor product of matrices or vector spaces over the complex numbers $\mathbb{C}$, while $\otimes$ denotes the fusion of excitations. Using

$$
R_{i}(g) \otimes_{\mathbb{C}} R_{j}(g)=\bigoplus_{k} N_{k}^{i j} R_{k}(g),
$$

we see that

$$
B_{i} B_{j}=\sum_{k} N_{k}^{i j} B_{k}
$$

The ends of the strings are pointlike topological excitations, and the above $N_{k}^{i j}$ are the fusion coefficients of those topological excitations. Let $d_{i}$ be the quantum dimension of those topological excitations that satisfy

$$
\sum_{j} N_{k}^{i j} d_{j}=d_{i} d_{k}
$$

and let

$$
B=\sum_{i} \frac{d_{i}}{D^{2}} B_{i}, \quad D^{2}=\sum_{i} d_{i}^{2}
$$

We have

$B^{2}=\sum_{i, j} \frac{d_{i} d_{j}}{D^{4}} B_{i} B_{j}=\sum_{i, j, k} \frac{d_{i} d_{j}}{D^{4}} N_{k}^{i j} B_{k}=\sum_{i, k} \frac{d_{i} d_{i}}{D^{4}} d_{k} B_{k}=B$.

Thus, $B$ is a projection operator. In fact, it is a projection operator into the subspace with $\prod_{I J \in \text { string }} g_{I J}=1$.

\section{Membrane operators}

A membrane is formed by the faces of the dual lattice, which is also a cubic lattice. The faces of the dual lattice correspond to the links in the original lattice and are also labeled by $I J$.

A membrane operator is given by

$$
Q_{a}=\sum_{h \in \chi_{a}} \prod_{I J \in \text { membrane }} \hat{A}_{I J}(h)
$$

where the operator $\hat{A}_{I J}(h)$ is defined as

$$
\hat{A}_{I J}(h)\left|g_{I J}\right\rangle=\left|h g_{I J}\right\rangle,
$$

and $\chi_{a}$ is the $a$ th conjugacy class of $G$. Here, $I$ 's are on one side of the membrane, and $J$ 's are on the other side of the membrane.
We note that

$$
Q_{a} Q_{b}=\sum_{h \in \chi_{a}} \sum_{\sim h \in \chi_{b}} \prod_{I J \in \text { membrane }} \hat{A}_{I J}(h \sim h)=\sum_{c} M_{c}^{a b} Q_{c} .
$$

The above expression allows us to see that $M_{c}^{a b}$ are nonnegative integers. Clearly, $Q_{a} Q_{b}=Q_{b} Q_{a}$ and $\left(Q_{a} Q_{b}\right) Q_{c}=$ $Q_{a}\left(Q_{b} Q_{c}\right)$, which implies that

$$
M_{c}^{a b}=M_{c}^{b a}, \quad \sum_{d} M_{d}^{a b} M_{e}^{d c}=\sum_{d} M_{e}^{a d} M_{d}^{b c} .
$$

Let $\left(M_{a}\right)_{c b}=M_{c}^{a b}$, and we can rewrite the second equation in the above as

$$
M_{c} M_{a}=M_{a} M_{c}
$$

For example, the permutation group of three elements $S_{3}=$ $\{(123),(132),(321),(213),(231),(312)\}$ has three conjugacy classes: $\chi_{1}=\{(123)\}, \chi_{2}=\{(132),(321),(213)\}$, and $\chi_{3}=\{(231),(312)\}$. We find that

$$
\begin{array}{lc}
Q_{1} Q_{a}=Q_{a}, & Q_{2} Q_{2}=3 Q_{1}+3 Q_{3}, \\
Q_{3} Q_{3}=2 Q_{1}+Q_{3}, & Q_{2} Q_{3}=2 Q_{2} .
\end{array}
$$

Let $\mathbf{c}$ be a common eigenvector of $M_{a}$ whose components are all non-negative. (Such a common eigenvector exists since the matrix elements of $M_{a}$ are all nonnegative.) The eigenvalue of such an eigenvector is $\lambda_{a}$ for $M_{a}$. We choose the scaling factor of $\mathbf{c}$ to satisfy

$$
\sum_{a} \lambda_{a} c_{a}=1
$$

In this case,

$$
Q^{2}=Q, \quad Q=\sum_{a} c_{a} Q_{a}
$$

\section{Commuting-projector Hamiltonian}

Let $Q_{I, a}$ be the smallest membrane operator that creates a small membrane corresponding to the surface of a cube in the dual lattice. Such a membrane wraps a site $I$ in the original cubic lattice. We note that $Q_{I, a}$ is a sum of gauge transformation operators $g_{I J} \rightarrow h G_{I J}$. Since the string operators are gauge invariant, we have

$$
\left[B_{i}, Q_{I, a}\right]=0
$$

Therefore, we can construct the following commutingprojector Hamiltonian $[62,63]$ : 


$$
H=\sum_{I}\left(1-Q_{I}\right)+\sum_{\langle I J K L\rangle}\left(1-B_{\langle I J K L\rangle}\right),
$$

where

$$
Q_{I}=\sum_{a} c_{a} Q_{I, a}, \quad B_{\langle I J K L\rangle}=\sum_{i} \frac{d_{i}}{D} B_{\langle I J K L\rangle, i}
$$

and $\langle I J K L\rangle$ labels the loops around the squares of the original cubic lattice.

The ground state of the above exactly soluble Hamiltonian has a nontrivial topological order. The lowenergy effective theory is the $G$-gauge theory.

\section{Pointlike and stringlike excitations}

What are the excitations for the above Hamiltonian? There are local pointlike excitations created by local operators, and there are also topological pointlike excitations that cannot be created by local operators. Two topological pointlike excitations are said to be equivalent if they differ by local pointlike excitations. The equivalent topological pointlike excitations are said to have the same type.

The different types of topological pointlike excitations are created at the ends of the open string operators that we discussed before. Thus, we see that types of topological pointlike excitations correspond one-to-one to the irreducible representations of $G$. In other words, topological pointlike excitations are described by $\mathcal{R} \operatorname{ep}(G)$ in a $G$-gauge theory.

Similarly, there are also topological stringlike excitations. They are created at the boundary of the open membrane operators. However, the types of membrane operators are not in one-to-one correspondence with the types of stringlike excitations. There are pure stringlike excitations, which correspond one-to-one to the conjugacy classes of $G$. There are also mixed stringlike excitations, which are bound states of pure stringlike excitations and pointlike excitations $[20,46,64]$. In general, the types (pure and mixed) of stringlike excitations in a $G$-gauge theory are labeled by a pair $\chi, R\left(G_{\chi}\right)$, where $\chi$ is a conjugacy class of $G, R\left(G_{\chi}\right)$ is a representation of $G_{\chi}$, and $G_{\chi}$ is a subgroup of $G$ whose elements all commute with a fixed element in $\chi$ (a centralizer subgroup).

For example, in $S_{3}$-gauge theory, the $\chi_{2}$-flux loop breaks the $S_{3}$ gauge "symmetry" down to $Z_{2}$ gauge symmetry. Thus, there are two types of $\chi_{2}$-flux-loop excitations - one carries no $Z_{2}$ charge (which is the pure one denoted by $s_{20}$ ), and the other carries $Z_{2}$ charge 1 (denoted by $s_{21}$ ). Similarly, the $\chi_{3}$-flux loop breaks the $S_{3}$ gauge symmetry down to $Z_{3}$ gauge symmetry. Therefore, there are three types of $\chi_{3}$-flux-loop excitations, carrying $Z_{3}$ charge $q=0,1,2$ (denoted by $s_{3 q}$ ). Thus, the string excitations in $S_{3}$-gauge theory are given by $s_{20}=\left(\chi_{2}, R_{0}\left(Z_{2}\right)\right), s_{21}=$ $\left(\chi_{2}, R_{1}\left(Z_{2}\right)\right), \quad s_{30}=\left(\chi_{3}, R_{0}\left(Z_{3}\right)\right), \quad s_{31}=\left(\chi_{3}, R_{1}\left(Z_{3}\right)\right)$, $s_{32}=\left(\chi_{3}, R_{2}\left(Z_{3}\right)\right)$. (Note that $G_{\chi_{2}}=Z_{2}$ and $G_{\chi_{3}}=Z_{3}$.) Those stringlike excitations also have a shrinking rule: If we shrink a string to a point, it will behave like a pointlike excitation:

$$
\begin{array}{ll}
s_{20} \rightarrow p_{0} \oplus p_{2}, & s_{21} \rightarrow p_{1} \oplus p_{2}, \\
s_{30} \rightarrow p_{0} \oplus p_{1}, & s_{31} \rightarrow p_{2}, \quad s_{32} \rightarrow p_{2} .
\end{array}
$$

In general, the string excitations whose shrinking rule contains the trivial excitation $p_{0}$ are the pure string excitations, which are $s_{20}$ and $s_{30}$ in the $S_{3}$-gauge example. We see that the types of pure string excitations are labeled by $\chi$, the conjugacy classes of $G$.

The $\chi_{1}$-flux loop (i.e., trivial flux loop) does not break the $S_{3}$ gauge. Thus, there are three types of $\chi_{1}$-fluxloop excitations-those carrying a trivial representation (denoted by $s_{10}$ or $p_{0}$ ), a nontrivial 1D irreducible representation (denoted by $s_{11}$ or $p_{1}$ ), or a $2 \mathrm{D}$ irreducible representation (denoted by $s_{12}$ or $p_{2}$ ) of $S_{3}$. In fact, those $\chi_{1}$-flux-loop excitations (or trivial-string excitations) correspond to the pointlike excitations. The fusions of all the stringlike excitations are given in Table III.

TABLE III. Fusion rules of pointlike and stringlike excitations in $3+1 \mathrm{D} S_{3}$ gauge theory. Here, $p_{0}$ corresponds to the trivial pointlike

\begin{tabular}{|c|c|c|c|c|c|c|c|c|}
\hline$\otimes$ & $p_{0}$ & $p_{1}$ & $p_{2}$ & $s_{20}$ (pure) & $s_{21}$ & $s_{30}$ (pure) & $s_{31}$ & $s_{32}$ \\
\hline$p_{0}$ & $p_{0}$ & $p_{1}$ & $p_{2}$ & $s_{20}$ & $s_{21}$ & $s_{30}$ & $s_{31}$ & $s_{32}$ \\
\hline$p_{1}$ & $p_{1}$ & $p_{0}$ & $p_{2}$ & $s_{21}$ & $s_{20}$ & $s_{30}$ & $s_{31}$ & $s_{32}$ \\
\hline$p_{2}$ & $p_{2}$ & $p_{2}$ & $p_{0} \oplus p_{1} \oplus p_{2}$ & $s_{20} \oplus s_{21}$ & $s_{20} \oplus s_{21}$ & $s_{31} \oplus s_{32}$ & $s_{30} \oplus s_{32}$ & $s_{30} \oplus s_{31}$ \\
\hline$s_{20}$ & $s_{20}$ & $s_{21}$ & $s_{20} \oplus s_{21}$ & $p_{0} \oplus p_{2} \oplus s_{30} \oplus s_{31} \oplus s_{32}$ & $p_{1} \oplus p_{2} \oplus s_{30} \oplus s_{31} \oplus s_{32}$ & $s_{20} \oplus s_{21}$ & $s_{20} \oplus s_{21}$ & $s_{20} \oplus s_{21}$ \\
\hline$s_{21}$ & $s_{21}$ & $s_{20}$ & $s_{20} \oplus s_{21}$ & $p_{1} \oplus p_{2} \oplus s_{30} \oplus s_{31} \oplus s_{32}$ & $p_{0} \oplus p_{2} \oplus s_{30} \oplus s_{31} \oplus s_{32}$ & $s_{20} \oplus s_{21}$ & $s_{20} \oplus s_{21}$ & $s_{20} \oplus s_{21}$ \\
\hline$s_{30}$ & $s_{30}$ & $s_{30}$ & $s_{31} \oplus s_{32}$ & $s_{20} \oplus s_{21}$ & $s_{20} \oplus s_{21}$ & $p_{0} \oplus p_{1} \oplus s_{30}$ & $s_{32} \oplus p_{2}$ & $s_{31} \oplus p_{2}$ \\
\hline$s_{31}$ & $s_{31}$ & $s_{31}$ & $s_{30} \oplus s_{32}$ & $s_{20} \oplus s_{21}$ & $s_{20} \oplus s_{21}$ & $s_{32} \oplus p_{2}$ & $p_{0} \oplus p_{1} \oplus s_{31}$ & $s_{30} \oplus p_{2}$ \\
\hline$s_{32}$ & $s_{32}$ & $s_{32}$ & $s_{30} \oplus s_{31}$ & $s_{20} \oplus s_{21}$ & $s_{20} \oplus s_{21}$ & $s_{31} \oplus p_{2}$ & $s_{30} \oplus p_{2}$ & $p_{0} \oplus p_{1} \oplus s_{32}$ \\
\hline
\end{tabular}
excitations and also to the trivial stringlike excitations. Note that $p_{1}$ and $p_{2}$ are nontrivial pointlike excitations corresponding to the $1 \mathrm{D}$ and $2 \mathrm{D}$ representations of $S_{3}$ (i.e., the charged particles). Also, $s_{20}$ and $s_{30}$ correspond to pure stringlike excitations labeled by conjugacy classes $\chi_{2}$ and $\chi_{3}$, and $s_{21}, s_{31}$, and $s_{32}$ are charge and string bound states, as one can see from the fusion rules. See Ref. [38]. 
We may regard looplike excitations $(\chi, q)$ with the same conjugacy class $\chi$ but different representations $q$ as equivalent and introduce the notion of pure type: The looplike excitation $(\chi, q)$ is said to have a pure type $\chi$. Thus, the fusion of the membrane operators corresponds to the fusion of pure types, which is closely related to the fusion of stringlike excitations, after we quotient out the $G_{\chi}$ representations $q$, by identifying

$$
\begin{aligned}
& p_{0}=Q_{1}, \quad p_{1}=Q_{1}, \quad p_{2}=2 Q_{1}, \\
& s_{2 q}=Q_{2}, \quad s_{3 q}=Q_{3},
\end{aligned}
$$

in the fusion rule in Table III. The general identification formula is

$$
s_{\chi q}=\operatorname{dim}(q) Q_{\chi},
$$

where $\operatorname{dim}(q)$ is the dimension of the $G_{\chi}$ representations $q$.

\section{APPENDIX B: GENERAL DISCUSSION OF STRING AND MEMBRANE OPERATORS IN 3+1D TOPOLOGICAL ORDERS}

\section{String operators in $3+1 \mathrm{D}$ topological orders}

For a generic $3+1 \mathrm{D}$ topological order, the type- $i$ particlelike excitations are still described by string operators

$$
B_{i}=\sum_{a_{1} a_{2} a_{3} \ldots} \hat{O}_{i}^{a a_{1}}\left(I_{1}\right) \hat{O}_{i}^{a_{1} a_{2}}\left(I_{2}\right) \hat{O}_{i}^{a_{2} a_{3}}\left(I_{3}\right) \ldots
$$

Here, the string operators are matrix product operators. The $\hat{O}_{i}^{a a_{1}}(I)$ are operators acting on the degrees of freedom on site $I$. We may view the superscripts $a, a_{1}, a_{2}, \ldots$ as the indices of the matrix elements for an operator-valued matrix. We say that two closed string operators are equivalent if they differ by a local unitary transformation. More precisely, two closed string operators are equivalent iff they can deform into each other while all the local operators maintain shortranged correlations. We also normalize the string operators such that $\left\langle B_{i}\right\rangle$ is independent of string length when the string is closed. Such normalized string operators satisfy the following fusion algebra:

$$
B_{i} B_{j}=N_{k}^{i j} B_{k} .
$$

We can show that $N_{k}^{i j}$ are non-negative integers by viewing the string operators as the world-lines in the time direction.

\section{Membrane operators in 3+1D topological orders}

Similarly, the type- $a$ stringlike excitations are described by membrane operators

$$
Q_{a}=\sum_{\left\{a_{I}\right\}} \hat{O}_{a}^{a_{1} a_{2} a_{3} a_{4}}\left(I_{1}\right) \hat{O}_{a}^{a_{4} a_{5} a_{6} a_{7}}\left(I_{2}\right) \hat{O}_{a}^{a_{3} a_{8} a_{9} a_{10}}\left(I_{3}\right) \ldots,
$$

which are tensor network operators. Here, $\hat{O}_{a}(I)$ are operator-valued tensors, and the superscripts are tensor indices. In addition, two closed membrane operators are equivalent if they differ by a local unitary transformation. More precisely, two closed membrane operators are equivalent iff they can deform into each other while all the local operators maintain short-ranged correlations.

The equivalent classes of membrane operators can be different for membrane operators with different topology. For example, the equivalent classes of the spherical closed membrane operators $Q_{a}^{S^{2}}$ correspond to the pure membrane types. The pure membrane type corresponds to the type for pure stringlike excitations. For toric closed membrane operators $Q_{a}^{T^{2}}$, the number of the equivalent classes will, in general, be different from the number of the equivalent classes of spherical closed membrane operators. This is because toric closed membrane operators may contain closed string operators wrapping around the noncontractible loops, which generate different equivalent classes. Clearly, if we do not have nontrivial pointlike excitations, then there will be a one-to-one correspondence between the spherical membrane operators and toric membrane operators.

Since the loop of the string operator on $S^{2}$ is always contractible, the spherical membrane operators do not contain loops of the string operator. Thus, the spherical membrane operators are labeled by the conjugacy classes only. The spherical membrane operators $Q_{\chi}^{S^{2}}$ also satisfy the following fusion algebra:

$$
Q_{\chi_{1}}^{S^{2}} Q_{\chi_{2}}^{S^{2}}=\sum_{k} M_{S^{2} \chi_{3}}^{\chi_{1} \chi_{2}} Q_{\chi_{3}}^{S^{2}}
$$

In particular,

$$
M_{S^{2} \chi_{3}}^{\chi_{1} \chi_{2}}=M_{\chi_{3}}^{\chi_{1} \chi_{2}},
$$

which can be calculated from the fusion of the conjugacy classes $\chi_{1}$ and $\chi_{2}$ [see Eq. (A11)].

\section{APPENDIX C: MORE GENERAL PROPERTIES OF STRINGLIKE EXCITATIONS IN 3 + 1D TOPOLOGICAL ORDERS}

\section{Pure stringlike excitations and sectors in dimension reduction}

In Sec. III B, we have shown that in $3+1 \mathrm{D}$, the number of sectors, $N_{1}^{\mathrm{sec}}$, in the dimension reduction is the number of classes of stringlike topological excitations that can be distinguished by the braiding with the pointlike excitations. However, in Sec. IV B, we have shown that all stringlike topological excitations can be distinguished from each other via their braiding properties with the pointlike excitations. Therefore, 
$N_{1}^{\mathrm{sec}}$ is the number of types of pure stringlike topological excitations, if all the pointlike excitations are bosons.

Let $\operatorname{GSD}_{\mathcal{C}^{d+1}}\left(M_{\text {space }}^{d}\right)$ be the ground-state degeneracy of a $d+1 \mathrm{D}$ topological order $\mathcal{C}^{d+1}$ on a closed $d$-dimensional space manifold $M_{\text {space }}^{d}$. We note that

$$
\operatorname{GSD}_{C^{d+1}}\left(S_{\text {space }}^{d}\right)=1
$$

Now, let us consider a more general dimension reduction where we reduce $d$-dimensional space $M_{\text {space }}^{d}=L_{\text {space }}^{d-n} \times S^{n}$ to $(d-n)$-dimensional space $L_{\text {space }}^{d-n}$ by shrinking the $S^{n}$. Here, $N_{n}^{\mathrm{sec}}$ is the number of sectors of the dimensionreduced topological orders. We find that

$$
N_{n}^{\mathrm{sec}}=\mathrm{GSD}_{\mathcal{C}^{d+1}}\left(S^{d-n} \times S^{n}\right) .
$$

We see that

$$
N_{n}^{\mathrm{sec}}=N_{d-n}^{\mathrm{sec}}
$$

\section{Pure stringlike excitations are labeled by the conjugacy classes of $G$}

From the last section, we see that for $3+1 \mathrm{D} A B$ topological order,

$$
N_{1}^{\mathrm{sec}}=N_{2}^{\mathrm{sec}}=\text { number of types of pure strings. }
$$

In the following, we show that

$N_{2}^{\mathrm{sec}}=$ number of types of pointlike excitations.

We consider the GSD on $S^{1} \times S^{2}$. We note that the path integral on space-time $S^{1} \times D^{3}$ gives us a particular ground state on $S^{1} \times S^{2}$. To obtain other ground states on $S^{1} \times S^{2}$, we insert a type- $i$ string operator $B_{i}$ along the $S^{1}$ in $S^{1} \times D^{3}$. The string operator is inserted at a particular point on $D^{3}$. The insertion of different string operators generate linear independent states. This is because the pointlike excitations represented by the string operators have nondegenerate braiding with the pure stringlike excitations.

The braiding between the inserted pointlike excitations and the stringlike excitations is described by creating a small loop of strings on $S^{2}=\partial D^{3}$. Then, we enlarge the loop and let the loop wrap around $S^{2}$. Such a braiding process is equivalent to applying the sphere membrane operator $Q_{a}$ on $S^{2}=\partial D^{3}$. The eigenvalues of the sphere membrane operators $Q_{a}$ should distinguish all the states created by inserting the string operators $B_{i}$. Therefore, the number of sectors, $N_{2}^{\mathrm{sec}}$, in the $S^{2}$-dimension reduction of a $3+1 \mathrm{D}$ topological order is the number of types of pointlike topological excitations.

This allows us to show that

for 3 + 1D topological orders, the number of types of pointlike excitations is the same as the number of types of pure stringlike excitations.

Moreover, from the fact that the untwisted sector of dimension reduction is the Drinfeld center $Z[\mathcal{R} \operatorname{ep}(G)]$, we know that

the pure stringlike excitations in generic $3+1 \mathrm{D} A B$ topological orders are labeled by the conjugacy classes of $G$.

Now, the dimension reduction of a generic bosonic $3+1 \mathrm{D}$ topological order $\mathcal{C}^{4}$ can be written as

$$
\mathcal{C}^{4}=\bigoplus_{\chi} \mathcal{C}_{\chi}^{3}
$$

where $\chi$ is the conjugacy class of the group $G$ whose representations form the $\mathrm{SFC}$ of $\mathcal{C}^{4}$, and $\sum_{\chi}$ sums over all the conjugacy classes of $G$.

From the dimension reduction Eq. (C6), we can also compute the ground-state degeneracy on the 3-torus,

$$
\operatorname{GSD}_{\mathcal{C}^{4}}\left(T^{3}\right)=\sum_{\chi} \operatorname{GSD}_{\mathcal{C}_{\chi}^{3}}\left(T^{2}\right)
$$

The degenerate ground states form a representation of the mapping class group of $T^{3}$, which is $S L(3, \mathbb{Z})$.

The dimensional reduction leads to the reduction of the representation of $S L(3, \mathbb{Z})$ to the representations of $S L(2, \mathbb{Z})$ that characterize the $2+1 \mathrm{D}$ dimension-reduced topological orders $\mathcal{C}_{\chi}^{3}$. We consider the $S L(2, \mathbb{Z}) \subset S L(3, \mathbb{Z})$ subgroup and the reduction of the $S L(3, \mathbb{Z})$ representation $R_{\mathcal{C}^{4}}$ to the $S L(2, \mathbb{Z})$ representations $R_{\mathcal{C}_{X}^{3}}$ :

$$
R_{\mathcal{C}^{4}}=\bigoplus_{\chi} R_{\mathcal{C}_{\chi}^{3}}
$$

The $S L(3, \mathbb{Z})$ representation $R_{\mathcal{C}^{4}}$ describes the $3+1 \mathrm{D}$ topological order $\mathcal{C}^{4}$, and the $S L(2, \mathbb{Z})$ representations $R_{\mathcal{C}_{\chi}^{3}}$ describe the $2+1 \mathrm{D}$ topological orders $\mathcal{C}_{\chi}^{3}$. The decomposition Eq. (C8) gives us the dimensional reduction Eq. (C6).

\section{Stringlike excitations are $G$ flux, even in generic 3+1D AB topological orders}

From the fact that the untwisted sector of dimension reduction is the Drinfeld center $Z[\mathcal{R e p}(G)]$, we know that 
the pure stringlike excitations in a generic $3+1 \mathrm{D} A B$ topological order have the same fusion ring as the gauge theory with the corresponding gauge group $G$.

Alternatively, we can argue the above claim using the results for the string-only boundary. This is because the bulk stringlike excitations can be obtained by lifting the boundary stringlike excitations. Since a bulk stringlike excitation $s_{i}$ can braid around a boundary stringlike excitation $s_{g}^{\text {bdry }}$, their fusion satisfies

$$
s_{i} \otimes s_{g}^{\text {bdry }}=s_{g}^{\text {bdry }} \otimes s_{i} .
$$

This result allows us to show that

$$
s_{i}=\bigoplus_{g \in \chi} s_{g}^{\text {bdry }} \equiv s_{\chi},
$$

where $\chi$ is a conjugacy class of $G$. Therefore, even in a generic $3+1 \mathrm{D}$ topological order, we may still view stringlike excitations as the $G$-gauge flux, which is described by the conjugacy classes of the group $G$. In particular, the bulk pure stringlike excitations fuse like the conjugacy classes [see Eq. (A11)]. As a result, the quantum dimension of a pure stringlike excitation is given by the size of the conjugacy class: $d_{s_{\chi}}=|\chi|$. This is one of the key results of this paper.

There is a simple physical way to understand the relation between the bulk and boundary stringlike excitations. Since the boundary is induced by the condensation of all the pointlike excitations, it corresponds to breaking all the "gauge symmetry." Thus, there is no gauge equivalence of $g \sim h g h^{-1}$, and there is no degeneracy between the flux loop that induces $g$ monodromy and $h g h^{-1}$ monodromy. Therefore, if we bring a bulk string $s_{\chi}$ near the boundary, it will split, $s_{\chi} \rightarrow \bigoplus_{g \in \chi} s_{g}^{\text {bdry }}$ [see Eq. (C10)].

\section{APPENDIX D: MORE ABOUT DIMENSION REDUCTION}

We argued that the stringlike excitations are $G$ flux, even in generic $3+1 \mathrm{D} \mathrm{AB}$ bosonic topological orders. Now, we can say more about the $2+1 \mathrm{D}$ topological orders $\mathcal{C}_{\chi}^{3}$ that appear in the dimension reduction Eq. (C6). We first note that the pointlike excitations in $3+1 \mathrm{D}$ topological order $\mathcal{C}^{4}$ are described by $\mathcal{R e p}(G)$ for a group $G$. In the dimension reduction, those $3+1 \mathrm{D}$ pointlike excitations become the $2+1 \mathrm{D}$ pointlike excitations with trivial mutual statistics between them; they form symmetric fusion subcategories $\mathcal{E}_{\chi}$ of the $2+1$ D dimension-reduced topological orders $\mathcal{C}_{\chi}^{3}$.

For the conjugacy class $\chi=\{1\}$, i.e., the untwisted sector, $\mathcal{E}_{\chi=\{1\}}=\mathcal{R e p}(G)$ and $\mathcal{C}_{\chi=\{1\}}^{3}=Z[\mathcal{R e p}(G)]$ is a minimal modular extension of $\mathcal{R} \operatorname{ep}(G)$. But what about the other conjugacy classes?

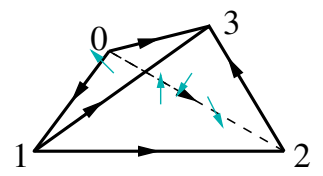

(a)

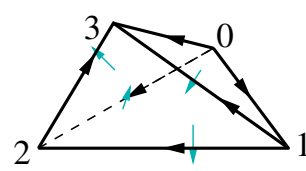

(b)
FIG. 10. Two branched simplices with opposite orientations. (a) A branched simplex with positive orientation. (b) A branched simplex with negative orientation.

Because $\mathcal{C}_{\chi}^{3}$ is induced by threading a $G$ flux described by $\chi$ through $S^{1}$, such a $G$ flux will break the gauge symmetry from $G$ to $G_{\chi}$, where $G_{\chi}$ is a subgroup of $G$ that commutes with a fixed element in conjugacy class $\chi$. Therefore, the $\operatorname{SFC} \mathcal{E}_{\chi}$ in $\mathcal{C}_{\chi}^{3}$ is given by $\mathcal{R e p}\left(G_{\chi}\right)$. The $3+1 \mathrm{D}$ pointlike excitations described by $\mathcal{R e p}(G)$ will split into $2+1 \mathrm{D}$ pointlike excitations described by $\operatorname{Rep}\left(G_{\chi}\right)$ in each sector (see Table II).

Similarly, for the other sectors, we have that

the dimension-reduced $2+1 \mathrm{D}$ topological orders $\mathcal{C}_{\chi}^{3}$ are minimal modular extensions of $\operatorname{Rep}\left(G_{\chi}\right)$.

The minimal modular extension means that the anyons in $\mathcal{C}_{\chi}^{3}$ that are not in $\operatorname{Rep}\left(G_{\chi}\right)$ all have nontrivial mutual statistics with the bosons in $\operatorname{Rep}\left(G_{\chi}\right)$. This condition comes from the result in Sec. IV B. Note that unlike the untwisted sector, $\mathcal{C}_{\chi}^{3}$, in general, is not the Drinfeld center of $\mathcal{R e p}\left(G_{\chi}\right)$.

\section{APPENDIX E: BRANCHING STRUCTURE OF SPACE-TIME LATTICE}

In order to define a generic lattice theory on the spacetime complex $M_{\text {latt }}^{d}$ using local tensors, it is important to give the vertices of each simplex a local order. A nice local scheme to order the vertices is given by a branching structure $[15,16,65]$. A branching structure is a choice of orientation of each link in the $d$-dimensional complex so that there is no oriented loop on any triangle (see Fig. 10).

The branching structure induces a local order of the vertices on each simplex. The first vertex of a simplex is the vertex with no incoming links, and the second vertex is the vertex with only one incoming link, etc. So the simplex in Fig. 10(a) has the following vertex ordering: $0,1,2,3$.

The branching structure also gives the simplex (and its subsimplices) a canonical orientation. Figure 10 illustrates two 3-simplices with opposite canonical orientations compared with the three-dimensional space in which they are embedded. The blue arrows indicate the canonical orientations of the 2-simplices. The black arrows indicate the canonical orientations of the 1 -simplices. 
[1] L. D. Landau, Theory of Phase Transformations I, Phys. Z. Sowjetunion 11, 26 (1937).

[2] L. D. Landau and E. M. Lifschitz, Statistical PhysicsCourse of Theoretical Physics (Pergamon, London, 1958), Vol. 5.

[3] X. Chen, Z.-C. Gu, and X.-G. Wen, Classification of Gapped Symmetric Phases in 1D Spin Systems, Phys. Rev. B 83, 035107 (2011).

[4] N. Schuch, D. Perez-Garcia, and I. Cirac, Classifying Quantum Phases Using Matrix Product States and PEPS, Phys. Rev. B 84, 165139 (2011).

[5] F. Pollmann, E. Berg, A. M. Turner, and M. Oshikawa, Symmetry Protection of Topological Phases in OneDimensional Quantum Spin Systems, Phys. Rev. B 85, 075125 (2012).

[6] Z.-C. Gu and X.-G. Wen, Tensor-Entanglement-Filtering Renormalization Approach and Symmetry Protected Topological Order, Phys. Rev. B 80, 155131 (2009).

[7] X.-G. Wen, Topological Orders in Rigid States, Int. J. Mod. Phys. B 04, 239 (1990).

[8] X.-G. Wen and Q. Niu, Ground State Degeneracy of the FQH States in the Presence of Random Potentials and on High Genus Riemann Surfaces, Phys. Rev. B 41, 9377 (1990).

[9] E. Keski-Vakkuri and X.-G. Wen, Ground State Structure of Hierarchical QH States on Torus and Modular Transformation, Int. J. Mod. Phys. B 07, 4227 (1993).

[10] E. Rowell, R. Stong, and Z. Wang, On Classification of Modular Tensor Categories, Commun. Math. Phys. 292, 343 (2009).

[11] X.-G. Wen, A Theory of $2+1 \mathrm{~d}$ Bosonic Topological Orders, Natl. Sci. Rev. 3, 68 (2016).

[12] T. Lan, L. Kong, and X.-G. Wen, A Theory of $2+1 \mathrm{D}$ Fermionic Topological Orders and Fermionic/Bosonic Topological Orders with Symmetries, Phys. Rev. B 94, 155113 (2016).

[13] M. Barkeshli, P. Bonderson, M. Cheng, and Z. Wang, Symmetry, Defects, and Gauging of Topological Phases, arXiv: 1410.4540.

[14] T. Lan, L. Kong, and X.-G. Wen, Classification of $(2+1)-$ Dimensional Topological Order and Symmetry-Protected Topological Order for Bosonic and Fermionic Systems with On-Site Symmetries, Phys. Rev. B 95, 235140 (2017).

[15] X. Chen, Z.-C. Gu, Z.-X. Liu, and X.-G. Wen, Symmetry Protected Topological Orders and the Group Cohomology of Their Symmetry Group, Phys. Rev. B 87, 155114 (2013).

[16] X. Chen, Z.-C. Gu, Z.-X. Liu, and X.-G. Wen, Symmetry Protected Topological Orders in Interacting Bosonic Systems, Science 338, 1604 (2012).

[17] M. Levin and Z.-C. Gu, Braiding Statistics Approach to Symmetry-Protected Topological Phases, Phys. Rev. B 86, 115109 (2012).

[18] B. Zeng and X.-G. Wen, Gapped Quantum Liquids and Topological Order, Stochastic Local Transformations and Emergence of Unitarity, Phys. Rev. B 91, 125121 (2015).

[19] B. Swingle and J. McGreevy, Renormalization Group Constructions of Topological Quantum Liquids and Beyond, Phys. Rev. B 93, 045127 (2016).

[20] L. Kong and X.-G. Wen, Braided Fusion Categories, Gravitational Anomalies, and the Mathematical Framework for Topological Orders in Any Dimensions, arXiv:1405 .5858 .

[21] L. Kong, X.-G. Wen, and H. Zheng, Boundary-Bulk Relation in Topological Orders, Nucl. Phys. B922, 62 (2017).

[22] V. G. Turaev and O. Y. Viro, State Sum Invariants of 3-Manifolds and Quantum 6j-Symbols, Topology 31, 865 (1992).

[23] M. Levin and X.-G. Wen, String-Net Condensation: A Physical Mechanism for Topological Phases, Phys. Rev. B 71, 045110 (2005).

[24] C. Chamon, Quantum Glassiness in Strongly Correlated Clean Systems: An Example of Topological Overprotection, Phys. Rev. Lett. 94, 040402 (2005).

[25] S. Bravyi, B. Leemhuis, and B. M. Terhal, Topological Order in an Exactly Solvable 3D Spin Model, Ann. Phys. (Amsterdam) 326, 839 (2011).

[26] S. Vijay, J. Haah, and L. Fu, Fracton Topological Order, Generalized Lattice Gauge Theory, and Duality, Phys. Rev. B 94, 235157 (2016).

[27] H. Ma, E. Lake, X. Chen, and M. Hermele, Fracton Topological Order via Coupled Layers, Phys. Rev. B 95, 245126 (2017).

[28] S. Vijay, Isotropic Layer Construction and Phase Diagram for Fracton Topological Phases, arXiv:1701.00762.

[29] T. H. Hsieh and G. B. Halász, Fractons from Partons, Phys. Rev. B 96, 165105 (2017).

[30] K. Slagle and Y. B. Kim, Fracton Topological Order from Nearest-Neighbor Two-Spin Interactions and Continuous Subdimensional Quantum Phase Transitions via Dualities, Phys. Rev. B 96, 165106 (2017).

[31] J. Haah, Local Stabilizer Codes in Three Dimensions without String Logical Operators, Phys. Rev. A 83, 042330 (2011).

[32] In this paper, we also call those pointlike excited states carrying reducible group representations (composite) pointlike excitations; in general, they have accidental degeneracy. This is unconventional in the physics literature but beneficial when we discuss the universal properties, such as fusion, of topological orders. The composite (accidentally degenerate) excitations also play an important role.

[33] This is true even for the general cases when some quasiparticles are fermions.

[34] R. Dijkgraaf and E. Witten, Topological Gauge Theories and Group Cohomology, Commun. Math. Phys. 129, 393 (1990).

[35] T. Lan and X.-G. Wen, A Classification of 3 + 1D Bosonic Topological Orders (II): The Case When Some Point-like Excitations Are Fermions, arXiv:1801.08530.

[36] M. Levin, Protected Edge Modes without Symmetry, Phys. Rev. X 3, 021009 (2013).

[37] X.-G. Wen, Classifying Gauge Anomalies through SPT Orders and Classifying Gravitational Anomalies through Topological Orders, Phys. Rev. D 88, 045013 (2013).

[38] H. Moradi and X.-G. Wen, Universal Topological Data for Gapped Quantum Liquids in Three Dimensions and Fusion Algebra for Non-Abelian String Excitations, Phys. Rev. B 91, 075114 (2015).

[39] J.C. Wang and X.-G. Wen, Non-Abelian String and Particle Braiding in Topological Order-Modular SL $(3, z)$ 
Representation and $(3+1)$-Dimensional Twisted Gauge Theory, Phys. Rev. B 91, 035134 (2015).

[40] A. Kapustin, Bosonic Topological Insulators and Paramagnets: A View from Cobordisms, arXiv:1404.6659.

[41] D. S. Freed, Short-Range Entanglement and Invertible Field Theories, arXiv:1406.7278.

[42] X.-G. Wen, Vacuum Degeneracy of Chiral Spin State in Compactified Spaces, Phys. Rev. B 40, 7387 (1989).

[43] V. Drinfeld, S. Gelaki, D. Nikshych, and V. Ostrik, On Braided Fusion Categories I, arXiv:0906.0620.

[44] It is similar in $2+1 \mathrm{D}$ topological orders. The topological $S$ matrix is the invariant of braiding, but it is the expectation value of double braidings, i.e., moving one particle a whole loop around another.

[45] N. Gurski, An Algebraic Theory of Tricategories (unpublished).

[46] D. V. Else and C. Nayak, Cheshire Charge in $(3+1)-D$ Topological Phases, Phys. Rev. B 96, 045136 (2017).

[47] L. Kong, Anyon Condensation and Tensor Categories, Nucl. Phys. B886, 436 (2014).

[48] A. Kirillov, Jr. and V. Ostrik, On q-Analog of McKay Correspondence and ADE Classification of $\mathrm{si}(2)$ Conformal Field Theories, arXiv:math/0101219.

[49] T. Lan, L. Kong, and X.-G. Wen, Modular Extensions of Unitary Braided Fusion Categories and $2+1 \mathrm{D}$ Topological/SPT Orders with Symmetries, Commun. Math. Phys. 351, 709 (2017).

[50] M. A. Levin and X.-G. Wen, String-Net Condensation: A Physical Mechanism for Topological Phases, Phys. Rev. B 71, 045110 (2005).

[51] T. Lan and X.-G. Wen, Topological Quasiparticles and the Holographic Bulk-Edge Relation in 2+1D String-Net Models, Phys. Rev. B 90, 115119 (2014).

[52] X.-G. Wen, Construction of Bosonic Symmetry-ProtectedTrivial States and Their Topological Invariants via $G \times$ SO $(\infty)$ Non-linear $\sigma$-Models, Phys. Rev. B 91, 205101 (2015).
[53] A. Vishwanath and T. Senthil, Physics of Three-Dimensional Bosonic Topological Insulators: Surface Deconfined Criticality and Quantized Magnetoelectric Effect, Phys. Rev. X 3, 011016 (2013).

[54] X.-G. Wen, Symmetry-Protected Topological Invariants of Symmetry-Protected Topological Phases of Interacting Bosons or Fermions, Phys. Rev. B 89, 035147 (2014).

[55] L.-Y. Hung and X.-G. Wen, Universal Symmetry-Protected Topological Invariants for Symmetry-Protected Topological States, Phys. Rev. B 89, 075121 (2014).

[56] J. C. Wang, Z.-C. Gu, and X.-G. Wen, Field-Theory Representation of Gauge-Gravity Symmetry-Protected Topological Invariants, Group Cohomology, and Beyond, Phys. Rev. Lett. 114, 031601 (2015).

[57] X.-G. Wen, Exactly Soluble Local Bosonic Cocycle Models, Statistical Transmutation, and Simplest Time-Reversal Symmetric Topological Orders in 3 + 1D, Phys. Rev. B 95, 205142 (2017).

[58] K. Walker and Z. Wang, $(3+1)$-TQFTs and Topological Insulators, arXiv:1104.2632.

[59] C. W. von Keyserlingk, F. J. Burnell, and S. H. Simon, Three-Dimensional Topological Lattice Models with Surface Anyons, Phys. Rev. B 87, 045107 (2013).

[60] D. J. Williamson and Z. Wang, Hamiltonian Realizations of (3+1)-TQFTs, Ann. Phys. (Amsterdam) 377, 311 (2017).

[61] X. Chen, F. J. Burnell, A. Vishwanath, and L. Fidkowski, Anomalous Symmetry Fractionalization and Surface Topological Order, Phys. Rev. X 5, 041013 (2015).

[62] A. Yu. Kitaev, Fault-Tolerant Quantum Computation by Anyons, Ann. Phys. (Amsterdam) 303, 2 (2003).

[63] A. Mesaros and Y. Ran, A Classification of Symmetry Enriched Topological Phases with Exactly Solvable Models, Phys. Rev. B 87, 155115 (2013).

[64] C. Wang and M. Levin, Braiding Statistics of Loop Excitations in Three Dimensions, Phys. Rev. Lett. 113, 080403 (2014).

[65] F. Costantino, A Calculus for Branched Spines of 3-Manifolds, Math. Z. 251, 427 (2005). 